\title{
Correspondances modulaires et les fonctions $\zeta$ de courbes algébriques.
}

\author{
Par Gorô SHIMURA
}

(Reçu le 5 Nov., 1957)

M. Eichler [3] a découvert qu'il y a une relation étroite entre l'opérateur $T_{p}$ défini par E. Hecke et les fonctions $\zeta$ de certains corps de fonctions modulaires elliptiques qui sont en rapport avec les formes quadratiques. Dans ce travail nous allons généraliser cette relation.

Soient $N$ un entier positif et $\mathfrak{M}_{N}$ le corps complet des fonctions modu laires elliptiques d'espèce ("Stufe») $N ; \mathfrak{M}_{N}$ est un corps de fonctions algébriques d'une variable sur le corps des nombres complexes $\boldsymbol{C}$. Nous pouvons montrer qu'il y a un sous-corps $\Re$ ayant le corps des nombres rationnels $\boldsymbol{Q}$ comme corps de constantes, qui engendre $\mathfrak{M}_{N}$ sur $\boldsymbol{C}$. Nous allons démontrer que les fonctions $\zeta$ d'un tel corps $\Re$, choisi convenablement, et de certains sous-corps de $\Re$ sont représèntées explicitement par la fonction $\zeta$ de Riemann et les produits d'Euler introduits par E. Hecke et que les valeurs absolues des racines caractéristiques de l'opérateur $T_{p}$ de Hecke pour les formes paraboliques ("Spitzenformen») de degré 2 ne dépassent pas $2 \sqrt{p}$ pour presque tous les nombres premiers $p$.

Nous démontrerons ces résultats en établissant deux formules de congruence ((I) et (II) dans $\S 3$ ) pour les correspondances modulaires. Nous représentons d'abord le corps $\mathfrak{M}_{N}$ par les coordonnées des points $t$ tels que $N t=0$ sur une courbe elliptique $E$ dont l'invariant est transcendant sur $\boldsymbol{Q}$. L'opérateur $T_{p}$ de Hecke peut être regardé comme la différentielle d'une correspondance algébrique que nous pouvons définir d'une manière algébrogéométrique par un homomorphisme $\lambda$ de $E$ tel que $\nu(\lambda)=p$ et que nous appelons la correspondance modulaire de degré $p$. Dans la théorie de la multiplication complexe ([6], [7]), la relation de congruence ou la décomposition de l'homomorphisme $\pi$ a été obtenue par la réduction d'homomorphismes d'une variété abélienne modulo un diviseur premier du corps de base. Une méthode analogue est employée ici pour démontrer la formule (I); nous considèrerons la réduction de l'homomorphisme $\lambda$ modulo un diviseur premier. Seulement nous nous occupons au cas présent d'une courbe elliptique sans multiplication complexe, mais on pourrait considérer $\lambda$ comme une "multiplication générique» de la courbe elliptique. La formule com- 
plémentaire (II) est démontrée au moyen du fait qui s'exprime dans la prop. $7(\S 2)$. Le résultat pour les racines caractéristiques de l'opérateur $T_{p}$ est une conséquence de la formule (I).

\section{$\S 1$. Courbes elliptiques.}

1. Nous désignerons par $\boldsymbol{Z}, \boldsymbol{Q}$ et $\boldsymbol{C}$ respectivement l'anneau des entiers rationnels, le corps des nombres rationnels et le corps des nombres complexes.

Soit $A$ une variété abélienne ${ }^{1)}$. Nous désignerons par $\mathcal{A}(A)$ l'anneau des endomorphismes de $A$, par $\mathcal{A}_{0}(A)$ le produit tensoriel $\mathcal{A}(A) \times \boldsymbol{Q}$ et par $\delta_{A}$ l'élément unité de $\mathcal{A}(A)$. Soient $B$ une autre variété abélienne de même dimension que $A$ et $\lambda$ un homomorphisme de $A$ sur $B$. Soient $k$ un corps de définition pour $A, B$ et $\lambda ; x$ un point générique de $A$ par rapport à $k$. Nous poserons

$$
\begin{gathered}
\nu(\lambda)=\left[k(x): k(\lambda(x)], \quad \nu_{s}(\lambda)=[k(x): k(\lambda x)]_{s},\right. \\
\nu_{i}(\lambda)=[k(x): k(\lambda x)]_{i} .
\end{gathered}
$$

Ces entiers ne dépendent que de $A, B$ et $\lambda$ et non du choix de $k$ et de $x$. Nous désignerons par $\mathrm{g}(\lambda)$ le noyau de $\lambda$ et $\operatorname{par} \mathfrak{g}(n, A)$ le noyau de $n \delta_{A}$ pour chaque entier $n$. Le groupe $g(\lambda)$ est d'ordre $\nu_{s}(\lambda)$. Si $A$ est de dimension $d$, on a $\nu\left(n \delta_{A}\right)=n^{2 d}$; par suite $\mathrm{g}(n, A)$ est d'ordre $n^{2 d}$ si $n$ n'est pas multiple de la caractéristique de $k$ [11]]). Pour le cas où $n$ est la caractéristique de $k$, on a le lemme suivant dont la démonstration est donnée dans [8].

Lеммe 1. Soient $k$ un corps de caractéristique $p \neq 0$ et $A$ une variété abélienne de dimension d définie par rapport à $k$. On a alors

$$
\nu_{i}\left(p \delta_{A}\right) \geqq p^{d}, \quad \nu_{s}\left(p \delta_{A}\right) \leqq p^{d} .
$$

Soient $k$ un corps de caractéristique $p \neq 0, q=p^{f}$ une puissance de $p$, où $f$ est un entier positif ou négatif. Nous désignerons par $k^{q}$ le corps des $q$-ièmes puissances des éléments de $k$. En faisant correspondre $z \in k$ à $z^{q} \in k^{q}$ on obtient un isomorphisme $\sigma$ de $k$ sur $k^{q}$. Soient $V$ une variété définie par rapport à $k$ et $x$ un point de $V$. Nous désignerons par $V^{q}$ et par $x^{q}$ la variété $V^{\sigma}$ transformée par $\sigma$ et le point de $V^{q}$ dont les coordonnées sont les $q$-ièmes puissances de celles de $x$. Soient $h$ une application rationnelle de $V$ dans une variété $W$, définie par rapport à $k$, et $H$ le graphe de $h$. Nous désignerons par $h^{q}$ l'application de $V^{q}$ dans $W^{q}$ dont le graphe est [11].

1) Il sera constamment fait usage des définitions et des résultats de [9], [10], 
$H^{q}$. Soient $A$ une variété abélienne définie par rapport à $k$ et $O$ l'élément neutre de $A$. Nous entendrons par la notation $A^{q}$ toujours la variété abélienne dont l'élément neutre est $O^{q}$. Si $\lambda$ est un homomorphisme de $A$ dans une variété abélienne, défini par rapport à $k, \lambda^{q}$ est un homomorphisme de $A^{q}$. Soit $x$ un point générique de $A$ par rapport à $k ; x^{q}$ est alors un point générique de $A^{q}$ par rapport à $k$. Comme on a $k(x) \supset k\left(x^{q}\right)$ on obtient une application rationnelle $\pi$ de $A$ sur $A^{q}$ telle que $\pi x=x^{q} ; \pi$ est un homomorphisme de $A$ sur $A^{q}$; on a $\pi t=t^{q}$ pour tout point $t$ de $A$. Nous appellerons $\pi$ l'homomorphisme de q-ième puissance de $A$. Si $A$ est de dimension $d$, on a $\nu(\pi)=\nu_{i}(\pi)=q^{d}$.

Lemme 2. Soient $A, B, C$ trois variétés abéliennes de même dimension, $\lambda$ un homomorphisme de $A$ sur $B$ et $\mu$ un homomorphisme de $A$ sur $C$. Supposons que $\mathcal{A}(A)$ soit isomorphe à $Z$ et que l'on ait $\nu(\lambda)=\nu(\mu), \nu_{i}(\lambda)=\nu_{i}(\mu)=1$. Alors, pour que $B$ soit isomorphe à $C$, il faut et il suffit qu'on ait $\mathfrak{g}(\lambda)=\mathfrak{g}(\mu)$.

D'après le th. 17 de [11] $\mathrm{n}^{\circ} 34$, si l'on a $\mathfrak{g}(\lambda)=\mathfrak{g}(\mu), B$ est isomorphe à $C$. Réciproquement supposons qu'il existe un isomorphisme $\eta$ de $B$ sur $C$. D'après le th. 27 de [11] n ${ }^{\circ} 52$, il y a un homomorphisme $\alpha$ de $C$ sur $A$. $\alpha \eta \lambda$ et $\alpha \mu$ sont contenus dans $\mathcal{H}(A)$. Comme $\mathcal{H}(A)$ est isomorphe à $\boldsymbol{Z}$, il y a deux entiers $n, n^{\prime}$ autres que 0 tels que $n \alpha \eta \lambda=n^{\prime} \alpha \mu$. On a alors $\nu\left(n \delta_{A}\right) \nu(\alpha) \nu(\eta) \nu(\lambda)=\nu\left(n^{\prime} \delta_{A}\right) \nu(\alpha) \nu(\mu)$. Si l'on désigne par $d$ la dimension de $A$, on a $\nu\left(n \delta_{A}\right)=n^{2 d}, \nu\left(n^{\prime} \delta_{A}\right)=n^{\prime 2 d}$. Comme $\nu(\eta)=1$ et $\nu(\lambda)=\nu(\mu)$, on a $n= \pm n^{\prime}$ et par conséquent $\eta \lambda= \pm \mu$; d'où résulte $g(\lambda)=\mathfrak{g}(\eta \lambda)=\mathfrak{g}(\mu)$.

Lemme 3. Les notations $A, B, C, \lambda, \mu$ et les hypothèses étant celles $d u$ lemme 2 , soient $k$ un corps de définition pour $A, B, C, \lambda, \mu$ et $\sigma$ un isomorphisme de $k$ tel que $A^{\sigma}=A, B^{\sigma}=C$. On a alors $\lambda^{\sigma}= \pm \mu$.

D'après le lemme 2, on a $\mathrm{g}\left(\lambda^{\sigma}\right)=\mathrm{g}(\mu)$. Il y a donc un automorphisme $\varepsilon$ de $C$ tel que $\lambda^{\sigma}=\varepsilon \mu$ en vertu du th. 17 de [11] $\mathrm{n}^{\circ} 34$. Comme $C$ est isogène à $A, \mathcal{A}(C)$ est isomorphe à $\boldsymbol{Z}$; on en déduit que $\varepsilon= \pm 1$; ce qui prouve notre lemme.

2. Une variété abélienne de dimension 1 est une courbe algébrique de genre 1. Réciproquement, une courbe algébrique de genre 1, définie par rapport à un corps $k$, ayant un point rationnel par rapport à $k$ a une structure de variété abélienne définie par rapport à $k$. Ci-après, par une courbe elliptique définie par rapport à $k$, nous entendrons une variété abélienne de dimension 1 définie par rapport à $k$.

Soit $k$ un corps dont la caractéristique n'est ni 2 ni 3. Soient $\gamma_{2}, \gamma_{3}$, deux éléments de $k$ tels que $\gamma_{2}{ }^{3}-27 \gamma_{3}{ }^{2} \neq 0$ et $E_{1}$ la courbe définie par l'équation

$$
X_{0} X_{2}^{2}=4 X_{1}^{3}-\gamma_{2} X_{0}^{2} X_{1}-r_{3} X_{0}^{3}
$$


dans le plan projectif. En prenant le point $\left(X_{0}, X_{1}, X_{2}\right)=(0,0,1)$ pour l'élément neutre de variété de groupe, nous pouvons considérer $E_{1}$ comme une variété abélienne définie par rapport à $k$. En posant $X=X_{1} / X_{0}$ et $Y=X_{2} / X_{0}$ dans l'équation (1), on a l'équation affine

$$
Y^{2}=4 X^{3}-r_{2} X-r_{3}
$$

Nous conviendrons d'entendre par la courbe elliptique définie par l'équation (2) la courbe elliptique projective définie par l'équation (1), ayant le point $(0,0,1)$ comme l'élément neutre 0 . La correspondance $(X, Y) \rightarrow(X,-Y)$ donne l'endomorphisme -1 de la courbe elliptique.

Soit $E$ une courbe elliptique définie par rapport à $k$. Il est bien connu que $E$ est isomorphe à une courbe elliptique définie par une équation $Y^{2}=4 X^{3}-\gamma_{2} X-\gamma_{3}$ où $\gamma_{2}, \gamma_{3}$ sont deux éléments de $k$. Nous appellerons $\gamma_{2}{ }^{3} /\left(\gamma_{2}{ }^{3}-27 \gamma_{3}{ }^{2}\right)$ l'invariant de $E$ et le désignerons par $j_{E}$ ou $j(E) ; j(E)$ est contenu dans tout corps de définition pour $E$. Pour que deux courbes elliptiques soient isomorphes, il faut et il suffit qu'elles aient le même invariant. Si $\sigma$ est un isomorphisme d'un corps de définition pour une courbe elliptique $E$, on a $j\left(E^{\sigma}\right)=j(E)^{\sigma}$.

Soient $E$ la courbe elliptique définie par l'équation (2) et $h$ la fonction sur $E$ qui prend les coordonnées aux points de $E$ (autrement dit, la fonction $X_{1} / X_{0}$ sur la courbe (1)). Nous appellerons $h$ la fonction canonique sur $E$. On voit que

$$
h(u)=h(v) \Leftrightarrow u= \pm v
$$

3. Soient $E$ une courbe elliptique et $\mathfrak{g}$ un sous-groupe fini de $E$. D'après le th. 17 de [11] $n^{\circ} 34$, il y a une courbe elliptique $E^{\prime}$ et un homomorphisme $\lambda$ de $E$ sur $E^{\prime}$ tel qu'on ait $\mathfrak{g}=\mathfrak{g}(\lambda), \nu_{i}(\lambda)=1$. D'après le même théorème, l'invariant $j\left(E^{\prime}\right)$ de $E^{\prime}$ ne dépend que de $E$, g et non du choix de $E^{\prime}$ et de $\lambda$. Nous désignerons $j\left(E^{\prime}\right)$ par $j(E / \mathfrak{g})$.

Proposition 1. Soient $E, E^{\prime}$ deux courbes elliptiques isogènes et $k_{0}$ le corps premier contenu dans un corps de définition pour $E, E^{\prime}$. Alors $j\left(E^{\prime}\right)$ est algébrique $\operatorname{sur} k_{0}\left(j_{E}\right)$.

D’après la définition d'isogénéité, il existe un homomorphisme $\lambda$ de $E$ sur $E^{\prime}$. Soient $k$ un corps de définition pour $E, E^{\prime}, \lambda$ et $x$ un point générique de $E$ par rapport à $k$; posons $q=\nu_{i}(\lambda)$. Alors il y a un sous-corps $K$ de $k(x)$ tel que $k(x)$ soit une extension purement inséparable de $K$ de degré $q$ et $K$ soit séparable sur $k(\lambda x)$. Comme $k(x)$ est de dimension 1 sur $k$, on a $K=k\left(x^{q}\right)$; par suite, en posant $\mu x^{q}=\lambda x$, on obtient un homomorphisme $\mu$ de $E^{q}$ sur $E^{\prime}$ défini par rapport à $k$, pour lequel on a $\nu_{i}(\mu)=1$. Soit $\sigma$ un isomorphisme du corps $k\left(j_{E}, j_{E^{\prime}}\right)$ fixant tous les éléments de $k_{0}\left(j_{E}\right) ; \mu^{\sigma}$ est 
alors un homomorphisme de $\left(E^{q}\right)^{\sigma}$ sur $E^{\prime \sigma}$. On a $j\left(E^{\prime \sigma}\right)=j\left(E^{\prime}\right)^{\sigma}$ et $j\left(E^{q \sigma}\right)$ $=\left(j(E)^{q}\right)^{\sigma}=j(E)^{q}$. Comme $E^{q \sigma}$ a l'invariant $j(E)^{q}=j\left(E^{q}\right)$, il y a un isomorphisme $\eta$ de $E^{q}$ sur $E^{q \sigma}$. $\mu^{\sigma} \eta$ est un homomorphisme de $E^{q}$ sur $E^{\prime \sigma}$ dont le noyau $g\left(\mu^{\sigma} \eta\right)$ est d'ordre $\nu(\mu)$. Comme on a $\nu_{i}\left(\mu^{\sigma} \eta\right)=1$, on a $j\left(E^{\prime \sigma}\right)=j\left(E^{q} / \mathrm{g}\left(\mu^{\sigma} \eta\right)\right)$. Il n'y a qu'un nombre fini de sous-groupes de $E^{q}$ ayant l'ordre $\nu(\mu)$; par suite il n'y a qu' un nombre fini de $j\left(E^{\prime}\right)^{\sigma}$; ce qui montre que $j\left(E^{\prime}\right)$ est algébrique sur $k_{0}\left(j_{E}\right)$.

Proposition 2. Soient $E, E^{\prime}$ deux courbes elliptiques définies par rapport à un corps de caractéristique $p \neq 0$ et $\lambda$ un homomorphisme de $E$ sur $E^{\prime}$ tel qu'on ait $\nu_{s}(\lambda)=1$. Alors $\nu(\lambda)$ est une puissance $q$ de $p$; il existe un isomorphisme $\varepsilon$ de $E^{q}$ sur $E^{\prime}$ tel qu'on ait $\lambda t=\varepsilon t^{q}$ pour tout $t \in E$.

Soient $k$ un corps de définition pour $E, E^{\prime}, \lambda$ et $x$ un point générique de $E$ par rapport à $k$. Comme $\nu_{s}(\lambda)=1, k(x)$ est purement inséparable sur $k(\lambda x)$; par suite $\nu(\lambda)=[k(x): k(\lambda x)]$ est une puissance $q$ de $p$. Comme $k(x)$ est de dimension 1 sur $k$, on a $k(\lambda x)=k\left(x^{q}\right)$. On obtient donc un isomorphisme $\varepsilon$ de $E^{q}$ sur $E^{\prime}$, défini par rapport à $k$, tel que $\varepsilon x^{q}=\lambda x$. On vérifie aisément $\varepsilon t^{q}=\lambda t$ pour tout $t \in E$.

Proposition 3. Soit E une courbe elliptique définie par rapport à un corps de caractéristique $p \neq 0$ telle qu'on ait $j(E)^{p^{2}} \neq j(E)$. On a alors $\nu_{i}\left(p \delta_{E}\right)=\nu_{s}\left(p \delta_{E}\right)=p$; $\mathrm{g}(p, E)$ est d'ordre $p$.

D'après le lemme 1 , on a $\nu_{i}\left(p \delta_{E}\right) \geqq p ; \nu_{i}\left(p \delta_{E}\right)$ est donc égal à $p$ ou $p^{2}$. Supposons qu'on ait $\nu_{i}\left(p \delta_{E}\right)=p^{2}$. Alors on a $\nu_{s}\left(p \delta_{E}\right)=1$. D'après la prop. 2, $E$ est isomorphe à $E^{p^{2}}$; par suite on a $j(E)=j\left(E^{p^{2}}\right)=j(E)^{p^{2}}$; ce qui est en contradiction avec l'hypothèse de la proposition. On a donc $\nu_{i}\left(p \delta_{E}\right)=p$ et par suite $\nu_{s}\left(p \delta_{E}\right)=p$, de sorte que $\mathrm{g}(p, E)$ est d'ordre $p$.

$\mathrm{P}_{\text {Roposition }} 4$. Soit $E$ une courbe elliptique telle que $j(E)$ soit transcendant sur le corps premier. $\mathcal{H}(E)$ est alors isomorphe $\grave{a} \boldsymbol{Z}$.

Cette proposition est bien connue au cas où la caractéristique du corps de base est 0 . Supposons donc que la caractéristique soit autre que 0 . $\mathscr{A}_{0}(E)$ est un corps (commutatif ou non-commutatif). D'après la prop. 3 et d'après le th. 14 de [11] $\mathrm{n}^{\circ} 31$, il existe un homomorphisme $\varphi$ de $\mathcal{H}_{0}(E)$ dans le corps des nombres $p$-adiques; $\varphi$ est un isomorphisme puisque $\mathcal{A}_{0}(E)$ est un corps. $\mathcal{A}_{0}(E)$ est donc un corps commutatif. On a $\left[\mathcal{A}_{0}(E): \boldsymbol{Q}\right] \leqq 2$ puisque, d'après le cor. 2 du th. 36 de [11] $n^{\circ} 69$, tout élément de $\mathcal{H}_{0}(E)$ satisfait à une équation à coefficients rationnels de degré 2 . Supposons que l'on ait $\left[\mathscr{A}_{0}(E): Q\right]=2$. Soit $l$ un nombre premier qui reste premier dans $\mathcal{A}_{0}(E)$. Soit g un sous-groupe de $E$ d'ordre $l$; Il y a une courbe elliptique $E^{\prime}$ et un homomorphisme $\lambda$ de $E$ sur $E^{\prime}$ tels que $g(\lambda)=g$. Pour un $l$ approprié, on peut facilement voir que $\mathcal{A}\left(E^{\prime}\right)$ n'est pas isomorphe à $\mathcal{A}(E)^{2)}$. D'autre

2) C'est une conséquence des résultats dans [8]. 
part, d'après la prop. 1 , si l'on désigne par $k_{0}$ le corps premier, $j(E)$ est algébrique sur $k_{0}\left(j\left(E^{\prime}\right)\right)$, de sorte que $j\left(E^{\prime}\right)$ est transcendant sur $k_{0}$; on peut en déduire que $\mathcal{A}\left(E^{\prime}\right)$ est isomorphe à $\mathcal{A}(E)$; ce qui est absurde. On a donc $\left[\mathcal{A}_{0}(E) ; \boldsymbol{Q}\right]=1$; par suite $\mathcal{H}(E)$ est isomorphe à $\boldsymbol{Z}$.

\section{§2. Correspondances modulaires.} l'équation

4. Soient $r$ une variable sur $\boldsymbol{Q}$ et $E$ la courbe elliptique définie par

$$
Y^{2}=4 X^{3}-\gamma X-\gamma
$$

Posons $j=j(E)$. On a alors $j=r /(\gamma-27), \gamma=27 j /(j-1)$ et $\boldsymbol{Q}(\gamma)=\boldsymbol{Q}(j) ; j$ est donc une variable sur $\boldsymbol{Q}$. Nous désignons par $\boldsymbol{F}$ la clôture algébrique du $\operatorname{corps} \boldsymbol{Q}(j)$. Soit $h$ la fonction canonique sur $E$. Dans cette section nous employons ces notations $E, r, j, h, \boldsymbol{F}$ toujours en ce sens.

Nous désignerons par $K_{N}{ }^{*}(E)$ et par $K_{N}(E)$, ou simplement par $K_{N}{ }^{*}$ et par $K_{N}$, respectivement les corps

$$
\begin{gathered}
\boldsymbol{Q}(j, t \mid t \in \mathfrak{g}(N, E)) \\
\text { et } \boldsymbol{Q}(j, h(t) \mid t \in \mathfrak{g}(N, E))
\end{gathered}
$$

pour chaque entier $N>0$. Ces corps sont galoisiens sur $\boldsymbol{Q}(j)$. Nous désignerons par $G_{N}(E)$ le groupe de Galois de $K_{N}(E)$ sur $\boldsymbol{Q}(j)$. Soit $\left\{t_{1}, t_{2}\right\}$ un système de générateurs de $\mathfrak{g}(N, E)$; on a alors

$$
\mathfrak{g}(N, E)=\left\{\alpha t_{1}+\beta t_{2} \mid \quad 0 \leqq \alpha<N, 0 \leqq \beta<N\right\} .
$$

Soit $\sigma$ un élément de $G_{N}(E) ; \sigma$ est prolongé à un automorphisme de $K_{N}{ }^{*}$ que nous désignons aussi par $\sigma .\left\{t_{1}^{\sigma}, t_{2}{ }^{\sigma}\right\}$ est un système de générateurs de $\mathrm{g}(N, E)$; il y a donc une matrice $\left(\begin{array}{ll}a & b \\ c & d\end{array}\right)$ à coefficients entiers telle qu'on ait

$$
\left(\begin{array}{c}
t_{1}^{\sigma} \\
t_{2}^{\sigma}
\end{array}\right)=\left(\begin{array}{ll}
a & b \\
c & d
\end{array}\right)\left(\begin{array}{c}
t_{1} \\
t_{2}
\end{array}\right)
$$

et par suite

$$
h\left(\alpha t_{1}+\beta t_{2}\right)^{\sigma}=h\left(\alpha^{\prime} t_{1}+\beta^{\prime} t_{2}\right), \quad\left(\alpha^{\prime} \beta^{\prime}\right)=\left(\begin{array}{ll}
\alpha & \beta
\end{array}\right)\left(\begin{array}{ll}
a & b \\
c & d
\end{array}\right) .
$$

L'automorphisme $\sigma$ est determiné par la matrice $\left(\begin{array}{ll}a & b \\ c & d\end{array}\right)$ et s'appellera l'automorphisme de $K_{N}(E)$ correspondant $\grave{a}\left(\begin{array}{ll}a & b \\ c & d\end{array}\right)$ par rapport $\grave{a}\left\{t_{1}, t_{2}\right\}$. Nous désignerons par $G_{N}{ }^{*}$ le groupe des matrices de degré 2 à coefficients dans l'anneau $\boldsymbol{Z} / N \boldsymbol{Z}$ dont les déterminants sont inversibles et par $S_{N}{ }^{*}$ le sousgroupe de $G_{N}{ }^{*}$ consistant en les éléments unimodulaires (c.- $\dot{a}-d$. de déterminant 
$=1)$. Nous désignerons respectivement par $G_{N}$ et $S_{N}$ les groupes $G_{N}{ }^{*} /\{ \pm I\}$ et $S_{N}{ }^{*} /\{ \pm I\}$. Comme ci-dessus nous pouvons faire correspondre à chaque $\sigma \in G_{N}(E)$, une matrice $\left(\begin{array}{ll}a & b \\ c & d\end{array}\right) \in G_{N}{ }^{*}$. Tenant compte de (3), on vérifie facilement que l'application $\sigma \rightarrow\left(\begin{array}{ll}a & b \\ c & d\end{array}\right)$ donne un isomorphisme de $G_{N}(E)$ dans $G_{N}=G_{N}^{*} /\{ \pm I\}$. Cet isomorphisme est surjectif :

Proposition 5. En faisant correspondre une matrice $\left(\begin{array}{ll}a & b \\ c & d\end{array}\right)$ à $\sigma \in G_{N}(E)$ par la relation (5), on obtient un isomorphisme de $G_{N}(E)$ sur $G_{N}$.

Soit $S_{N}(E)$ le sous-groupe de $G_{N}(E)$ correspondant au sous-groupe $S_{N}$ de $G_{N}$ par cet isomorphisme. $S_{N}(E)$ ne dépend pas du choix de $\left\{t_{1}, t_{2}\right\}$.

Proposition 6. Le sous-corps de $K_{N}$ correspondant au sous-groupe $S_{N}(E)$ de $G_{N}(E)$ est le corps $\boldsymbol{Q}\left(j, \zeta_{N}\right)$ où $\zeta_{N}$ désigne une racine primitive $N$-ième d'unité. $\boldsymbol{Q}\left(\zeta_{N}\right)$ est algébriquement fermé dans $K_{N}$.

Dans $\S \S 2,3$, nous désignerons par $\zeta_{N}$ une racine primitive $N$-ième d'unité. Proposition 7. Soit $\left(\begin{array}{ll}a & b \\ c & d\end{array}\right)$ un élément de $G_{N}{ }^{*}$ et $\sigma$ l'automorphisme de $K_{N}$ correspondant à $\left(\begin{array}{ll}a & b \\ c & d\end{array}\right)$. On a alors $\zeta_{N}{ }^{\sigma}=\zeta_{N}^{a d-b c}$. En d'autres termes, si $a d-b c$ est congru à un nombre premier p modulo $N,\left(\begin{array}{ll}a & b \\ c & d\end{array}\right)$ donne la substitution de Frobenius $\left(\frac{\boldsymbol{Q}\left(\zeta_{N}\right) / \boldsymbol{Q}}{p}\right)$ dans $\boldsymbol{Q}\left(\zeta_{N}\right)$.

Nous démontrerons les prop. 5-7 dans le $§ 4$.

5. Maintenant nous allons déterminer les sous-groupes de $G_{N}(E)$ correspondant à certains sous-corps de $K_{N}(E)$. Dans ce but nous fixons un système de générateurs $\left\{t_{1}, t_{2}\right\}$ de $\mathrm{g}(N, E)$ et identifions $G_{N}(E)$ avec $G_{N}$ par l'isomorphisme donné ci-dessus.

Proposition 8. Soient $\mathfrak{g}, \mathfrak{g}^{\prime}$ deux sous-groupes d'ordre $N$ de $E$ et $\sigma$ un automorphisme de $\boldsymbol{F}$ sur $\boldsymbol{Q}(j)$. Alors pour qu'on ait $j(E / \mathfrak{g})^{\sigma}=j\left(E / \mathfrak{g}^{\prime}\right)$, il faut et il suffit que $\mathfrak{g}^{\sigma}=\mathfrak{g}^{\prime} ; j(E / \mathfrak{g})$ est contenu dans $K_{N}(E)$.

La première assertion est une conséquence immédiate de la prop. 4 et du lemme 2. Si $\sigma$ est l'identité dans $K_{N}(E)$, on a $h\left(t^{\sigma}\right)=h(t)$ pour $t \in \mathfrak{g}(N, E)$; par suite on a $t^{\sigma}= \pm t$ pour $t \in \mathfrak{g}$, de sorte qu'on a $\mathrm{g}^{\sigma}=\mathrm{g}$ et $j(E / \mathfrak{g})^{\sigma}=j(E / \mathfrak{g})$. Il s'ensuit de là que $j(E / g)$ est contenu dans $K_{N}(E)$.

Soient $\mathfrak{g}_{(1)}, \mathfrak{g}_{(2)}$ respectivement les sous-groupes de $E$ engendrés par $t_{1}$ et par $t_{2}$; posons $j_{(1)}=j\left(E / g_{(1)}\right), j_{(2)} \doteq j\left(E / g_{(2)}\right)$. Nous avons le tableau suivant: 


$\begin{array}{ll}\text { Sous-corps de } K_{N}(E) & \text { Sous-groupe de } G_{N}(E) \\ \boldsymbol{Q}\left(j, j_{(1)}\right) & \left\{\left(\begin{array}{ll}a & 0 \\ c & d\end{array}\right)\right\} /\{ \pm I\} \\ \boldsymbol{Q}\left(j, j_{(2)}\right) & \left\{\left(\begin{array}{ll}a & b \\ 0 & d\end{array}\right)\right\} /\{ \pm I\} \\ \boldsymbol{Q}\left(j, h\left(t_{1}\right)\right) & \left\{\left(\begin{array}{rr} \pm 1 & 0 \\ c & d\end{array}\right)\right\} /\{ \pm I\} \\ \boldsymbol{Q}\left(j, h\left(t_{2}\right)\right) & \left\{\left(\begin{array}{ll}a & b \\ 0 & \pm 1\end{array}\right)\right\} /\{ \pm I\} \\ \boldsymbol{Q}\left(j, j_{(2)}, h\left(t_{1}\right)\right) & \left\{\left(\begin{array}{rr} \pm 1 & 0 \\ 0 & d\end{array}\right)\right\} /\{ \pm I\} \\ \boldsymbol{Q}\left(j, j_{(1)}, h\left(t_{2}\right)\right) & \left\{\left(\begin{array}{rr}a & 0 \\ 0 & \pm 1\end{array}\right)\right\} /\{ \pm I\}\end{array}$

où les groupes au côté droit désignent les sous-groupes de $G_{N}(E)$ correspondant aux sous-corps de $K_{N}(E)$ au côté gauche; les lettres $a, d$ désignent les éléments inversibles de $\boldsymbol{Z} / N \boldsymbol{Z}$ et $b, c$ désignent les éléments quelconques de $\boldsymbol{Z} / N \boldsymbol{Z}$. Soit en effet $\sigma$ un élément de $G_{N}(E)$ donné par une matrice $\left(\begin{array}{ll}a & b \\ c & d\end{array}\right)$; on a alors $h\left(t_{1}^{\sigma}\right)=h\left(a t_{1}+b t_{2}\right)$; d'où résulte $\pm t_{1}{ }^{\sigma}=a t_{1}+b t_{2}$. D'après la prop. 8, pour qu'on ait $j_{(1)}^{\sigma}=j_{(1)}$, il faut et il suffit qu'on ait $\mathfrak{g}_{1}{ }^{\sigma}=\mathfrak{g}_{1}$, ou qu'on ait $b=0$. De plus, pour qu'on ait $h\left(t_{1}\right)^{\sigma}=h\left(t_{1}\right)$, il faut et il suffit qu'on ait $a= \pm 1, b=0$. Nous obtenons le tableau par ces relations.

6. Soient maintenant $n$ un entier positif tel que $(n, N)=1$ et $\mathrm{g}_{\alpha}(1 \leqq \alpha \leqq s)$ les sous-groupes cycliques de $E$ d'ordre $n$; posons $j_{\alpha}=j\left(E / \mathfrak{g}_{\alpha}\right)$. D'après la prop. $1, j$ est algébrique sur $\boldsymbol{Q}\left(j_{\alpha}\right)$, de sorte que $j_{\alpha}$ est transcendant sur $\boldsymbol{Q}$ pour chaque $\alpha$. Il existe donc un isomorphisme $\tau_{\alpha}$ de $\boldsymbol{Q}(j)$ sur $\boldsymbol{Q}\left(j_{\alpha}\right)$ tel que $j^{\tau} \alpha=j_{\alpha}$ pour chaque $\alpha$. Posons $\gamma_{\alpha}=\gamma^{\tau_{\alpha}}, E_{\alpha}=E^{\tau_{\alpha}}, h_{\alpha}=h^{\tau_{\alpha}}(1 \leqq \alpha \leqq s) ; E_{\alpha}$ est alors défini par l'équation

$$
Y^{2}=4 X^{3}-r_{a} X-r_{a}
$$

$h_{\alpha}$ est la fonction canonique sur $E_{\alpha}$. Comme on a $j\left(E_{\alpha}\right)=j_{\alpha}=j\left(E / \mathfrak{g}_{\alpha}\right)$, il existe un homomorphisme $\lambda_{\alpha}$ de $E$ sur $E_{\alpha}$ tel que $\mathfrak{g}_{\alpha}=\mathfrak{g}\left(\lambda_{\alpha}\right)$ pour chaque $\alpha$.

Proposition 9. Il existe un isomorphisme $\sigma_{\alpha}$ de $K_{N}(E)$ sur $K_{N}\left(E_{\alpha}\right)$ tel que

$$
j^{\sigma} \alpha=j_{\alpha}, h(t)^{\sigma} \alpha=h_{\alpha}\left(\lambda_{\alpha} t\right) \text { pour } t \in \mathrm{g}(N, E)
$$

pour chaque $\alpha$; $\sigma_{\alpha}$ est déterminé par ces relations.

Prolongeons l'isomorphisme $\tau_{\alpha}$ à un automorphisme de $\boldsymbol{F}$ que nous désignons aussi par $\tau_{\alpha}$. L'application $t \rightarrow t^{\tau_{\alpha}-1}$ donne un isomorphisme de $\mathrm{g}\left(N, E_{\alpha}\right)$ sur $\mathrm{g}(N, E)$. D'autre part, comme $(n, N)=1$, l'application $t \rightarrow \lambda_{\alpha} t$ 
donne un isomorphisme de $\mathfrak{g}(N, E)$ sur $\mathfrak{g}\left(N, E_{\alpha}\right)$; par suite $t \rightarrow\left(\lambda_{\alpha} t\right)^{\tau} \alpha^{-1}$ donne un automorphisme de $\mathfrak{g}(N, E)$; il existe donc une matrice $\left(\begin{array}{ll}a & b \\ c & d\end{array}\right)$ à coefficients entiers telle que

$$
\left(\begin{array}{l}
\lambda_{\alpha} t_{1} \\
\lambda_{\alpha} t_{2}
\end{array}\right)^{\tau_{\alpha}-1}=\left(\begin{array}{ll}
a & b \\
c & d
\end{array}\right)\left(\begin{array}{c}
t_{1} \\
t_{2}
\end{array}\right)
$$

D'après la prop. 5 , il existe un automorphisme $\rho_{\alpha}$ de $K_{N}(E)$ donné par $\left(\begin{array}{ll}a & b \\ c & d\end{array}\right)$; on a alors $j^{n} \alpha=j, h(t)^{\rho_{\alpha} \alpha}=h\left(\left(\lambda_{\alpha} t\right)^{\left.\tau_{\alpha}{ }^{-1}\right)}\right.$. Posons $\sigma_{\alpha}=\rho_{\alpha} \tau_{\alpha}$; on a alors $j^{\sigma_{\alpha}}=j_{\alpha}, h(t)^{\sigma_{\alpha}}=h_{\alpha}\left(\lambda_{\alpha} t\right)$. Comme $j, h(t)$ engendrent $K_{N}(E)$ sur $\boldsymbol{Q}, \sigma_{\alpha}$ est déterminé par ces relations.

Proposition 10. Soit $u=\left(u_{1}, \cdots, u_{m}\right)$ un ensemble fini d'éléments de $K_{N}(E)$ tel que $j$ soit contenu dans $\boldsymbol{Q}(u)$. Soient $\sigma_{1}, \cdots, \sigma_{s}$ les isomorphismes de $K_{N}$ déterminés dans la prop. 9. Alors $\boldsymbol{Q}\left(u, j^{\sigma_{\alpha}}\right)$ contient $\boldsymbol{Q}\left(u^{\sigma_{\alpha}}\right) ;\left(u^{\sigma_{1}}, \cdots, u^{\sigma_{s}}\right)$ est l'ensemble complet des conjugués de $u^{\sigma 1}$ sur $\boldsymbol{Q}(u)$.

Soit $\tau$ un automorphisme de la clôture algébrique $\boldsymbol{F}$ de $\boldsymbol{Q}(j)$ qui fixe $u, j_{\alpha}$. Comme $j$ est contenu dans $\boldsymbol{Q}(u), \tau$ laisse fixe $j$; on a donc $E^{\tau}=E$, $E_{\alpha}{ }^{\tau}=E_{\alpha}$. D'après le lemme 3 , on a $\lambda_{\alpha}{ }^{\tau}= \pm \lambda_{\alpha}$. Il en résulte que

$$
h(t)^{\sigma} \alpha^{\tau}=h_{\alpha}\left(\lambda_{\alpha} t\right)^{\tau}=h_{\alpha}\left( \pm \lambda_{\alpha} t^{\tau}\right)=h_{\alpha}\left(\lambda_{\alpha} t^{\tau}\right)=h\left(t^{\tau}\right)^{\sigma_{\alpha}}=h(t)^{\tau \sigma_{\alpha}}
$$

pour chaque $t \in \mathfrak{g}(N, E)$. On vérifie aisément $j^{\sigma_{\alpha} \tau}=j_{\alpha}=j^{\tau \sigma_{\alpha}}$. Comme $j$ et $h(t)$ engendrent $K_{N}(E)$, on a $\sigma_{\alpha} \tau=\tau \sigma_{\alpha}$; par suite on a $\left(u^{\sigma_{\alpha}}\right)^{\tau}=\left(u^{\tau}\right)^{\sigma_{\alpha}}=u^{\sigma_{\alpha}}$; ce qui montre que $\boldsymbol{Q}\left(u^{\sigma} \alpha\right)$ est contenu dans $\boldsymbol{Q}\left(u, j_{\alpha}\right)$. On déduit de là

$$
\left[\boldsymbol{Q}\left(u, u^{\sigma_{\alpha}}\right): \boldsymbol{Q}(u)\right]=\left[\boldsymbol{Q}\left(u, j_{\alpha}\right): \boldsymbol{Q}(u)\right] \leqq\left[\boldsymbol{Q}\left(j, j_{\alpha}\right): \boldsymbol{Q}(j)\right] \text {. }
$$

Soit $\sigma$ un automorphisme de $\boldsymbol{F}$ sur $\boldsymbol{Q}(j) ; \mathfrak{g}_{1}{ }^{\sigma}$ est alors un des $g_{\alpha}$; par suite, d'après la prop. 8 , les conjugués de $j_{1}$ sur $\boldsymbol{Q}(j)$ sont contenus dans $\left\{j_{1}, \cdots, j_{s}\right\}$; ce qui montre $\left[\boldsymbol{Q}\left(j, j_{\alpha}\right): \boldsymbol{Q}(j)\right] \leqq s$. Comme on a $\boldsymbol{Q}\left(u^{\sigma} \alpha\right) \supset \boldsymbol{Q}\left(j^{\sigma} \alpha\right)$ et $j^{\sigma_{\alpha}} \neq j^{\sigma} \beta$ pour $\alpha \neq \beta, u^{\sigma_{1}}, \cdots, u^{\sigma_{s}}$ sont différents l'un de l'autre. Notre proposition sera donc démontrée si nous faisons voir que $u^{\sigma_{\alpha}}$ est un conjugué de $u^{\sigma_{1}}$ sur $\boldsymbol{Q}(u)$ pour chaque $\alpha$. Soit $\left\{t_{1}{ }^{\prime}, t_{2}{ }^{\prime}\right\}$ un système de générateurs de $g(n N, E) ;\left\{N t_{1}{ }^{\prime} N t_{2}{ }^{\prime}\right\}$ est alors un système de générateurs de $g(n, E)$. D’après la prop. 5 , il existe un automorphisme $\rho$ de $K_{n}^{*}(E)$ sur $\boldsymbol{Q}(j)$ tel que $\mathfrak{g}_{1}{ }^{\rho}=\mathfrak{g}_{\alpha}$. Soit $\left(\begin{array}{ll}\alpha & \beta \\ \gamma & \delta\end{array}\right)$ une matrice de $G_{n}{ }^{*}$ à laquelle $\rho$ correspond par rapport à $\left\{N t_{1}{ }^{\prime}, N t_{2}{ }^{\prime}\right\}$. Comme on a $(n, N)=1$, il existe une matrice $\left(\begin{array}{ll}a & b \\ c & d\end{array}\right)$ de $G_{n N}^{*}$ telle que

$$
\begin{aligned}
& \left(\begin{array}{ll}
a & b \\
c & d
\end{array}\right) \equiv\left(\begin{array}{ll}
\alpha & \beta \\
\gamma & \delta
\end{array}\right) \text { mod. } n, \\
& \left(\begin{array}{ll}
a & b \\
c & d
\end{array}\right) \equiv\left(\begin{array}{ll}
1 & 0 \\
0 & 1
\end{array}\right) \text { mod. } N .
\end{aligned}
$$


Désignons par $\sigma$ l'automorphisme de $K_{n N}(E)$ correspondant à $\left(\begin{array}{ll}a & b \\ c & d\end{array}\right)$ par rapport à $\left\{t_{1}{ }^{\prime}, t_{2}{ }^{\prime}\right\}$. On a alors $z^{\sigma}=z$ pour $z \in K_{N}(E)$ et $w^{\sigma}=w^{\rho}$ pour $w \in K_{n}(E)$; on a donc $E^{\sigma}=E, \mathfrak{g}_{1}{ }^{\sigma}=\mathfrak{g}_{\alpha}, E_{1}{ }^{\sigma}=E_{\alpha}, j_{1}{ }^{\sigma}=j_{\alpha}, h_{1}{ }^{\sigma}=h_{\alpha}, \lambda_{1}{ }^{\sigma}= \pm \lambda_{\alpha}$ en vertu du lemme 3 et $t^{\sigma}= \pm t$ pour $t \in \mathfrak{g}(N, E)$; il s'ensuit de là que, pour $t \in \mathfrak{g}(N, E)$, on a

$$
h(t)^{\sigma_{1} \sigma}=h_{1}\left(\lambda_{1} t\right)^{\sigma}=h_{\alpha}\left( \pm \lambda_{\alpha}( \pm t)\right)=h_{\alpha}\left(\lambda_{\alpha} t\right)=h(t)^{\sigma_{\alpha}}
$$

et $j^{\sigma_{1} \sigma}=j^{\sigma} \alpha$. Comme $j$ et $h(t)$ pour $t \in \mathfrak{g}(N, E)$ engendrent $K_{N}(E)$, on obtient $\sigma_{1} \sigma=\sigma_{\alpha}$ sur $K_{N}(E)$; on a donc $\left(u^{\sigma_{1}}\right)^{\sigma}=u^{\sigma_{\alpha}}$. Comme $\sigma$ est l'identité sur $K_{N}(E)$, $u^{\sigma_{\alpha}}$ est un conjugué de $u^{\sigma_{1}}$ sur $K_{N}(E)$; ceci achève la démonstration.

Proposítion 11. Les notations étant celles de la prop. 10, on a

$$
\left[\boldsymbol{Q}\left(u, u^{\sigma_{1}}\right): \boldsymbol{Q}(u)\right]=\left[\boldsymbol{Q}\left(u, u^{\sigma_{1}}\right): \boldsymbol{Q}\left(u^{\sigma_{1}}\right)\right] \text {. }
$$

D'après le th. 27 de [11] $\mathrm{n}^{\circ} 52$, il existe un homomorphisme $\mu$ de $E_{1}$ sur $E$ tel que $\mu \lambda_{1}=n \delta_{E}$. En appliquant la prop. 9 à $E_{1}, \mu, E$, on peut montrer qu'il y a un isomorphisme $\tau$ de $K_{N}\left(E_{1}\right)$ sur $K_{N}(E)$ tel que

$$
j_{1}^{\tau}=j, h_{1}\left(t_{1}\right)^{\tau}=h\left(\mu t_{1}\right) \text { pour } t_{1} \in \mathrm{g}\left(N, E_{1}\right) .
$$

On a alors $j^{\sigma_{1} \tau}=j, h(t)^{\sigma_{1} \tau}=h(n t)$ pour $t \in \mathfrak{g}(N, E)$; autrement dit, $\sigma_{1} \tau$ est l'élément de $G_{N}(E)$ correspondant à $\left(\begin{array}{ll}n & 0 \\ 0 & n\end{array}\right)$. $\sigma_{1} \tau$ est donc contenu dans le centre du groupe $G_{N}(E)$; il en résulte qu'on a $\boldsymbol{Q}\left(u^{\sigma_{1} \tau}\right)=\boldsymbol{Q}(u)$. D'après la prop. 10, on a $\left[\boldsymbol{Q}\left(u, u^{\sigma_{1}}\right): \boldsymbol{Q}(u)\right]=s=$ le nombre des sous-groupes cycliques de $E$ d'ordre $n$. En appliquant ce résultat à $u^{\sigma_{1}}, \tau$, on obtient $\left[\boldsymbol{Q}\left(u^{\sigma_{1}}, u^{\sigma_{1} \tau}\right): \boldsymbol{Q}\left(u^{\sigma_{1}}\right)\right]=s$; ce qui prouve la proposition.

7. Soit $L$ un sous-corps de $K_{N}(E)$ tel que $L \supset \boldsymbol{Q}(j), L \cap \boldsymbol{Q}\left(\zeta_{N}\right)=\boldsymbol{Q}$. Comme $\boldsymbol{Q}\left(\zeta_{N}\right)$ est algébriquement fermé dans $K_{N}, \boldsymbol{Q}$ est algébriquement fermé dans $L$. Il y a donc une courbe complète $\Gamma$ définie par rapport à $\boldsymbol{Q}$, sans point multiple, telle qu'on ait $L=\boldsymbol{Q}(u)$ pour un point générique $u$ de $\Gamma$ par rapport à $\boldsymbol{Q}$. Le système $\{\Gamma, u\}$ s'appellera un modèle de $L$. Nous fixons pour le moment $L$ et $\{\Gamma, u\}$. Nous allons définir maintenant certaines correspondances algébriques sur la courbe $\Gamma$.

Soient $\sigma_{1}$ l'isomorphisme de $K_{N}$ déterminé à la prop. 9 et $X_{n}$ le diviseur premier rationnel par rapport à $\boldsymbol{Q}$ sur $\Gamma \times \Gamma$ ayant $u \times u^{\sigma_{1}}$ comme point générique $\operatorname{sur} \boldsymbol{Q}$. D’après la prop. 10 et d'après le th. 12 de [9] chap. VIIIn on a

$$
X_{n} \cdot(u \times \Gamma)=u \times \sum_{\alpha=1}^{s} u^{\sigma} \alpha .
$$

$X_{n}$ s'appellera la correspondance modulaire de degré $n$ sur $\Gamma$. D'après la prop). 11 , on a 


$$
d\left(X_{n}\right)=d^{\prime}\left(X_{n}\right)=s^{3)} .
$$

Soit $\rho_{n}$ l'élément de $G_{N}(E)$ correspondant à la matrice $\left(\begin{array}{ll}n & 0 \\ 0 & n\end{array}\right)$. Il est clair que cet automorphisme de $K_{N}$ ne dépend pas du choix d'un système de générateurs pour $\mathrm{g}(N, E)$. Soit $Y_{n}$ le lieu de $u \times u^{\rho n}$ sur $\Gamma \times \Gamma$ par rapport à $\boldsymbol{Q}$. Comme $\left(\begin{array}{ll}n & 0 \\ 0 & n\end{array}\right)$ est contenu dans le centre du groupe $G_{N}^{*}, \rho_{n}$ donne un automorphisme de $L$; il en résulte que l'on a $\boldsymbol{Q}(u)=\boldsymbol{Q}\left(u^{\rho n}\right)$, de sorte que $Y_{n}$ donne une correspondance birationnelle de $\Gamma$. On a donc

$$
Y_{n}(u)=u^{\rho n} \text {. }
$$

Si $n \equiv n^{\prime} \bmod . N$, on a $Y_{n}=Y_{n^{\prime}}$.

8. En outre de $X_{n}, Y_{n}$ nous avons besoin d'une autre correspondance pour exprimer la formule de congruence (II); pour la définir nous nous bornons à considérer le cas particulier où le corps $L$ est explicitement donné ainsi qu'il suit.

Fixons un système de générateurs $\left\{t_{1}, t_{2}\right\}$ de $\mathfrak{g}(N, E)$ et identifions $G_{N}(E)$ avec $G_{N}$ au moyen de l'isomorphisme défini par $\left\{t_{1}, t_{2}\right\}$. Nous désignerons par $H_{N}$ le sous-groupe $\left\{\left(\begin{array}{rr}a & 0 \\ 0 & \pm 1\end{array}\right) \mid(a, N)=1\right\} /\{ \pm I\}$ de $G$ et par $L_{N}$ le souscorps de $K_{N}$ correspondant à $H_{N}$. On voit que

on a donc

$$
H_{N} S_{N}=G_{N}, \quad H_{N} \cap S_{N}=\{e\} ;
$$

$$
L_{N} \cap \boldsymbol{Q}\left(\zeta_{N}\right)=\boldsymbol{Q}, \quad L_{N}\left(\zeta_{N}\right)=K_{N} .
$$

D’après le tableau dans 5 , on a

$$
L_{N}=\boldsymbol{Q}\left(j, j_{(1)}, h\left(t_{2}\right)\right) \text {. }
$$

Nous fixons un modèle de $L_{N}$ et le désignons par $\left\{\Gamma_{N}, u\right\}$. Soit $\psi$ l'automorphisme de $K_{N}$ correspondant à $\left(\begin{array}{rr}0 & 1 \\ -1 & 0\end{array}\right)$ par rapport à $\left\{t_{1}, t_{2}\right\}$. Il est clair que $\boldsymbol{Q}\left(\zeta_{N}, u\right)=\boldsymbol{Q}\left(\zeta_{N}, u^{\psi}\right)=K_{N}$. Soit $A$ le lieu de $u \times u^{\psi}$ sur $\Gamma_{N} \times \Gamma_{N}$ par rapport à $\boldsymbol{Q}\left(\zeta_{N}\right) ; A$ donne une correspondance birationnelle de $\Gamma_{N}$; on a donc

$$
A(u)=u^{\psi} .
$$

$\mathrm{P}_{\mathrm{ROPOSITION}}$ 12. Soit $\varphi_{n}$ l'automorphisme de $\boldsymbol{Q}\left(\zeta_{N}\right)$ tel que $\zeta_{N}{ }^{\varphi_{n}}=\zeta_{N}{ }^{n}$. On a alors

$$
A^{\varphi n} \circ Y_{n}=A .
$$

Soient $\tau_{n}$ et $\tau_{n}^{\prime}$ les automorphismes de $K_{N}$ qui correspondent respective-

3) Pour les définitions des notations $d(X), d^{\prime}(X)$, voir [10] p. 31 . 
ment à $\left(\begin{array}{ll}1 & 0 \\ 0 & n\end{array}\right)$ et $\left(\begin{array}{ll}n & 0 \\ 0 & 1\end{array}\right)$; d'après la prop. 7 , on a $\tau_{n}=\tau_{n}{ }^{\prime}=\varphi_{n} \operatorname{sur} \boldsymbol{Q}\left(\zeta_{N}\right)$. On vérifie aisément $\psi \tau_{n}=\tau_{n}{ }^{\prime} \psi$ et $\tau_{n}{ }^{\prime} \tau_{n}=\rho_{n}$. Comme $\tau_{n}{ }^{\prime}$ est contenu dans $H_{N}$, on a

$$
\left(u \times u^{\psi}\right)^{\tau} n=u^{\tau} \times \times u^{\psi^{\tau} n}=u^{\tau n^{\prime} \tau} \times u^{\tau n^{\prime} \psi}=u^{\rho n} \times u^{\psi} ;
$$

$u^{\rho_{n}} \times u^{\psi}$ est donc un point générique de $A^{\varphi_{n}}$ sur $\boldsymbol{Q}\left(\zeta_{N}\right)$; d'où résultent les relations

Par suite on a

$$
A^{\prime p n}\left(u^{\natural n}\right)=u^{\psi}, \quad\left(A^{\varphi n}\right)^{\prime}\left(u^{\psi}\right)=u^{\rho n}
$$

$$
A^{\rho_{n}}\left[Y_{n}(u)\right]=u^{\psi}=A(u), Y_{n}^{\prime}\left[\left(A^{\varphi_{n}}\right)^{\prime}\left(u^{\psi}\right)\right]=u=A^{\prime}\left(u^{\psi}\right) .
$$

$A$ et $Y_{n}$ sont rationnels par rapport à $\boldsymbol{Q}\left(\zeta_{N}\right)$ et $u, u^{\psi}$ sont génériques sur $\Gamma$ par rapport à $\boldsymbol{Q}\left(\zeta_{N}\right)$. Il s'ensuit donc de la définition de $A^{\varphi_{n}} Y_{n}$ ([10] II, $\left.\S 1, \mathrm{n}^{\circ} 5\right)$ que $A^{\prime n} n_{\circ} Y_{n}=A$.

Soit $\mathfrak{h}$ un sous-groupe du groupe d'éléments inversibles de $\boldsymbol{Z} / N \boldsymbol{Z}$; nous supposons que $\mathfrak{h}$ contienne -1 . Nous désignerons par $H_{N, \mathfrak{h}}$ le sous-groupe $\left\{\left(\begin{array}{ll}a & b \\ 0 & d\end{array}\right) \mid(a, N)=1, d \in \mathfrak{h}\right\} /\{ \pm I\}$ de $G_{N}$ et par $M_{N, \mathfrak{b}}$ le sous-corps de $K_{N}$ cor-

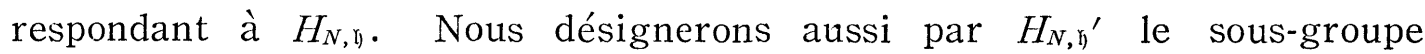
$\left\{\left(\begin{array}{ll}a & b \\ 0 & d\end{array}\right) \mid(d, N)=1, a \in \mathfrak{h}\right\} /\{ \pm I\}$ de $G_{N}$ et par $M_{N, \mathfrak{h}^{\prime}}$ le sous-corps de $K_{N}$ correspondant à $H_{N, \mathfrak{r}^{\prime}}$. On voit que

on a donc

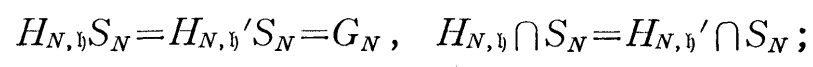

$$
M_{N, \mathfrak{h}} \cap \boldsymbol{Q}\left(\zeta_{N}\right)=M_{N, \mathfrak{h}^{\prime}} \cap \boldsymbol{Q}\left(\zeta_{N}\right)=\boldsymbol{Q}, \quad M_{N, \mathfrak{h}}\left(\zeta_{N}\right)=M_{N, \mathfrak{h}}{ }^{\prime}\left(\zeta_{N}\right) .
$$

Selon que $\mathfrak{h}$ ne contient que \pm 1 ou que $\mathfrak{h}$ contient tous les éléments inversibles de $\boldsymbol{Z} / N \boldsymbol{Z}$ nous désignerons $H_{N, \mathfrak{l},}, H_{N, \mathfrak{l}^{\prime}}, M_{N, \mathfrak{l},}, M_{N, \mathfrak{f}^{\prime}}$ respectivement par $H_{N, 1}$, $H_{N, 1}{ }^{\prime}, M_{N, 1}, M_{N, 1}{ }^{\prime}$ ou par $H_{N, 0}, H_{N, 0}{ }^{\prime}, M_{N, 0}, M_{N, 0}{ }^{\prime}$. On a alors

$$
\begin{aligned}
& H_{N, 0} \supset H_{N, \mathfrak{h}} \supset H_{N, 1}, \quad H_{N, 0}{ }^{\prime} \supset H_{N, \mathfrak{h}^{\prime}} \supset H_{N, 1^{\prime}}{ }^{\prime} \text {, } \\
& M_{N, 0} \subset M_{N, \mathfrak{h}} \subset M_{N, 1}, \quad M_{N, 0}{ }^{\prime} \subset M_{N, \mathfrak{l} \mathfrak{l}^{\prime}} \subset M_{N, 1}{ }^{\prime}
\end{aligned}
$$

pour chaque $\mathfrak{h}$; on voit aussi que $H_{N, 0}=H_{N, 0}{ }^{\prime}$ et $M_{N, 0}=M_{N, 0}{ }^{\prime}$.

Nous allons démontrer qu'il existe un isomorphisme de $M_{N, \mathfrak{h}}$ sur $M_{N, \mathfrak{h}}{ }^{\prime}$. $\left\{t_{1}, t_{2}\right\}$ étant le système de générateurs pour $\mathrm{g}(N, E)$ que nous avons fixé, nous désignons par $\mathfrak{g}$ le groupe engendré par $t_{2}$. Posons $j^{\prime}=j(E / \mathfrak{g})$. D'après la prop. $1, j^{\prime}$ est transcendant sur $\boldsymbol{Q}$ et $\boldsymbol{F}$ est la clôture algébrique de $\boldsymbol{Q}\left(j^{\prime}\right)$. Soit $\sigma$ un automorphisme de $\boldsymbol{F}$ tel que $j^{\sigma}=j^{\prime}$; posons $E^{\prime}=E^{\sigma}$. Il existe alors un homomorphisme $\lambda$ de $E$ sur $E^{\prime}$ tel que $\mathfrak{g}=\mathfrak{g}(\lambda)$. D'après le th. 27 de [11] $\mathrm{n}^{\circ} 52$, il existe un homomorphisme $\mu$ de $E^{\prime}$ sur $E$ tel que $\mu \lambda=N \delta_{E}, \lambda \mu=N \delta_{E^{\prime}}$. 
Posons $t_{1}{ }^{\prime}=\lambda t_{1}$. On voit que $\mu t_{1}{ }^{\prime}=0$ et que $t_{1}{ }^{\prime}$ est d'ordre $N$. Comme on a $\nu(\mu)=N, t_{1}{ }^{\prime}$ engendre $\mathrm{g}(\mu) . t_{2}{ }^{\sigma}$ est un point de $E^{\prime}$ d'ordre $N$; par suite, d'après la prop. 5 , il existe un automorphisme $\tau$ de $\boldsymbol{F}$ sur $\boldsymbol{Q}\left(j^{\prime}\right)$ tel que $\left(t_{2}{ }^{\sigma}\right)^{\tau}= \pm t_{1}{ }^{\prime}$; posons $\rho=\sigma \tau$; alors $\rho$ est un automorphisme de $\boldsymbol{F}$. On a $j^{\rho}=j^{\prime}$, $E^{o}=E^{\prime}, t_{2}{ }^{\rho}= \pm t_{1}{ }^{\prime}$, de sorte qu'on a $\mathfrak{g}\left(\lambda^{\rho}\right)=\mathfrak{g}(\lambda)^{\circ}=\mathfrak{g}(\mu)$; on en conclut que $E^{\prime \rho}$ est isomorphe à $E$; on a donc $j^{\prime \rho}=j$. En rappelant que $E$ est la courbe définie par l'équation (4), on voit que $E^{\prime o}=E^{\sigma \rho}$ est définie par l'équation

$$
Y^{2}=4 X^{3}-\gamma^{\sigma \rho} X-\gamma^{\sigma \rho} \text {. }
$$

On a $\gamma^{\sigma \rho}=27 j^{\sigma \rho} /\left(j^{\sigma o}-1\right)=27 j /(j-1)=r$; ce qui montre que $E^{\prime \rho}=E$. Soient $\alpha$ et $\alpha^{\prime}$ les automorphismes de $\boldsymbol{F}$ qui correspondent respectivement à $\left(\begin{array}{ll}a & b \\ 0 & d\end{array}\right)$ et $\left(\begin{array}{ll}d & b \\ 0 & a\end{array}\right)$ par rapport à $\left\{t_{1}, t_{2}\right\}$; on a alors $t_{1}{ }^{\alpha}= \pm\left(a t_{1}+b t_{2}\right)$ et ${t_{2}}^{\alpha^{\prime}}= \pm a t_{2}$. Nous allons maintenant montrer que $\rho \alpha=\alpha^{\prime} \rho$ sur $M_{N, 1}$. D'après le tableau dans 5, on a $M_{N, 1}=\boldsymbol{Q}\left(j, h\left(t_{2}\right)\right), M_{N, 0}=M_{N, 0}{ }^{\prime}=\boldsymbol{Q}\left(j, j^{\prime}\right)$. Comme $\alpha$ et $\alpha^{\prime}$ fixent les éléments de $M_{N, 0}$, on a $E^{\alpha}=E^{\alpha^{\prime}}=E, E^{\prime \alpha}=E^{\alpha^{\prime}}=E^{\prime}$. D'après le lemme 3 , on a $\lambda^{\alpha}= \pm \lambda, \lambda^{\alpha^{\prime}}= \pm \lambda$. On voit que $j^{\rho \alpha}=j^{\prime}=j^{\alpha^{\prime}, a}, h^{n \alpha}=h^{n}=h^{\alpha^{\prime} s}$ et

$$
\begin{aligned}
h\left(t_{2}\right)^{o \alpha} & =\left[h^{\rho}\left( \pm t_{1}{ }^{\prime}\right)\right]^{\alpha}=\left[h^{\rho}\left(\lambda t_{1}\right)\right]^{\alpha}=h^{\rho}\left(\lambda\left(\alpha t_{1}+b t_{2}\right)\right) \\
& =h^{\rho}\left(a \lambda t_{1}\right)=h^{\rho}\left( \pm \alpha t_{1}{ }^{\prime}\right)=h\left(a t_{2}\right)^{\rho}=h\left(t_{2}\right)^{\alpha \rho} .
\end{aligned}
$$

On obtient ainsi $j^{\rho \alpha}=j^{\alpha^{\prime} \rho}$ et $h\left(t_{2}\right)^{\rho \alpha}=h\left(t_{2}\right)^{\alpha^{\prime} \cdot \rho}$; il s'ensuit de là qu'on a $\rho \alpha=\alpha^{\prime} \rho$ sur $M_{N, 1}$. Si $a \in \mathfrak{h}, \alpha^{\prime}$ fixe les éléments de $M_{N, \mathfrak{h}}$; on a alors $\rho \alpha=\rho$ sur $M_{N, \mathfrak{h}}$; autrement dit, si $a \in \mathfrak{h}, \alpha$ fixe les éléments de $M_{N, \mathfrak{h}}{ }^{\circ}$; d'où résulte $M_{N, \mathfrak{h}}{ }^{\circ} \subset$ $M_{N, \mathfrak{h}^{\prime}}$. D'autre part, comme on a bien $M_{N, 0^{\circ}}=M_{N, 0}=M_{N, 0^{\prime}}$ et comme $\left[M_{N, \mathfrak{h}}\right.$ : $\left.M_{N, 0}\right],\left[M_{N, \mathfrak{h}^{\prime}}: M_{N, 0}{ }^{\prime}\right]$ sont égaux à l'indice du groupe $\mathfrak{h}$, on a

$$
\left[M_{N, \mathfrak{b}}{ }^{\rho}: M_{N, 0}\right]=\left[M_{N, \mathfrak{\natural}}: M_{N, 0}\right]=\left[M_{N, b^{\prime}}: M_{N, 0}\right] \text {; }
$$

ce qui montre $M_{N, f^{\rho}}=M_{N, r^{\prime}}$.

Soient maintenant $\left\{\Gamma_{N, \eta}, u\right\}$ un modèle du corps $M_{N, \eta}$ et $B$ le lieu de $u \times u^{\varrho}$ sur $\Gamma_{N, \mathfrak{G}} \times \Gamma_{N, \mathfrak{G}}$ par rapport à $\boldsymbol{Q}\left(\zeta_{N}\right)$, D'après la relation (11) et $M_{N, \mathfrak{f}}{ }^{\circ}$ $=M_{N, \mathfrak{h}}{ }^{\prime}, B$ donne une correspondance birationnelle de $\Gamma_{N, \mathfrak{h}}$. En vertu de la relation $\rho \alpha=\alpha^{\prime} \rho$ que l'on a vue ci-dessus, on peut démontrer la proposition suivante de la même manière que la prop. 12.

$\mathrm{P}_{\mathrm{ROPO}} \mathrm{stion}$ 13. Soit $\varphi_{n}$ l'automorphisme de $\boldsymbol{Q}\left(\zeta_{N}\right)$ tel que $\zeta_{N}{ }^{\varphi_{n}}=\zeta_{N}{ }^{n}$. On $a$ alors

$$
B^{\varphi} n_{\circ} Y_{n}=B
$$

\section{§ 3. Formules de congruence.}

9. Dans cette section nous démontrerons quelques relations de congruence pour les correspondances définies dans $\S 2$. Pour cela, nous profiterons 
de la notion de réduction des variétés algébriques modulo $p$, et nous servirons des termes et des résultats dans [5-8].

Soient $k$ un corps, $\mathfrak{p}$ un diviseur premier de $k$ et $\tilde{k}$ le corps des restes modulo $\mathfrak{p}$. Si $\mathfrak{X}$ représente un objet algébro-géométrique défini par rapport à $k$, nous indiquerons par $\mathfrak{X}(\mathfrak{p})$ ou $\dddot{\mathfrak{X}}$ la réduction de $\mathfrak{X}$ modulo $\mathfrak{p}$.

Soit $\Gamma$ une courbe complète sans point multiple, définie par rapport à $k$. Nous dirons que $\Gamma n^{\prime} a$ pas de défaut pour $\mathfrak{p}$, si $\Gamma$ est $\mathfrak{p}$-simple et $\mathfrak{p}$-complet et la réduction $\tilde{\Gamma}$ n'a pas de point multiple; s'il en est ainsi, $\Gamma$ et $\tilde{\Gamma}$ ont le même genre et $\Gamma$ n'a pas de défaut comme variété abélienne lorsque $\Gamma$ est une courbe elliptique.

Maitenant supposons que $\mathfrak{p}$ ne divise ni 2 , ni 3 . Soit $j$ un élément de $k$. Pour qu'il existe une courbe elliptique $E^{\prime}$, définie par rapport à une extension $k^{\prime}$ de $k$, sans défaut pour chaque prolongement de $\mathfrak{p}$ dans $k^{\prime}$, telle que $j\left(E^{\prime}\right)=j$, il faut et il suffit que $j$ soit p-entier; s'il en est ainsi, on a $j\left(E^{\prime}(\mathfrak{p})\right)$ $=j\left(E^{\prime}\right)\left(\mathfrak{p}^{\prime}\right)$ où $\mathfrak{p}^{\prime}$ désigne un prolongement de $\mathfrak{p}$ dans $k^{\prime}$ (cf. [2] $\S 4$ ).

Soient $\Gamma, \Gamma^{\prime}$ deux courbes complètes sans point multiple définies par rapport à $k$ et $X$ une correspondance entre $\Gamma$ et $\Gamma^{\prime}$ rationnelle par rapport à $k$. Supposons que $\Gamma, \Gamma^{\prime}$ n'aient pas de défaut pour $\mathfrak{p}$. On obtient alors une correspondance $\tilde{X}=X(p)$ entre $\tilde{\Gamma}$ et $\tilde{\Gamma}^{\prime}$; on vérifie aisément $X^{\prime}(p)=X(p)^{\prime}$. Soient $w$ et $\tilde{w}$ respectivement un point générique de $\Gamma$ par rapport à $k$ et un point générique de $\tilde{\Gamma}$ par rapport à $\tilde{k}$. Les produits $X \cdot\left(w \times \Gamma^{\prime}\right)$ et $\tilde{X} \cdot\left(\tilde{w} \times \tilde{\Gamma}^{\prime}\right)$ sont alors définis. La spécialisation $w \rightarrow \tilde{w}[p]$ définit un prolongement $\mathfrak{p}_{1}$ dans $k(w)$; d'après le th. 17 de [5], $\tilde{X} \cdot\left(\tilde{w} \times \tilde{\Gamma}^{\prime}\right)$ est la réduction de $X \cdot\left(w \times \Gamma^{\prime}\right)$ modulo $\mathfrak{p}_{1}$. Par suite on a $d(X)=d(\tilde{X})$ et semblablement $d^{\prime}(X)$ $=d^{\prime}(\tilde{X})$. Soient $J, J^{\prime}$ respectivement une jacobienne de $\Gamma$ et une jacobienne de $\Gamma^{\prime}$; soient $\varphi$ une application canonique de $\Gamma$ dans $J$ et $\varphi^{\prime}$ une application canonique de $\Gamma^{\prime}$ dans $J^{\prime}$. Nous supposons que $J, J^{\prime}, \varphi, \varphi^{\prime}$ soient définis par rapport à $k$ et que $J, J^{\prime}$ n'aient pas de défaut pour p. Alors, on peut démontrer que $J$ est une jacobienne de $\tilde{\Gamma}$, que $\tilde{\varphi}$ est une application canonique de $\tilde{\Gamma}$ dans $J$ et de même pour $\tilde{\Gamma}^{\prime}, \tilde{J}^{\prime}, \tilde{\varphi}^{\prime}$. Soit $\xi$ l'homomorphisme de $J$ dans $J^{\prime}$ correspondant à $X$. Alors on vérifie aisément que $\tilde{\xi}$ est l'homomorphisme de $J$ dans $J^{\prime}$ correspondant à $\tilde{X}$.

10. Soit maintenant $E$ la courbe elliptique avec l'invariant transcendant $j$ sur $\boldsymbol{Q}$, que nous avons considérée dans $\S 2$. Soient $N$ un entier positif et $p$ un nombre premier qui ne divise pas $6 N$. En regardant ce nombre premier $p$ comme le nombre $n$ dans 6 , nous obtenons les sous-groupes ga de $E$ d'ordre $p$, les courbes elliptiques $E_{\alpha}$ et les homomorphismes $\lambda_{\alpha}$. Le nombre $s$ des $g_{\alpha}$ est ici égal à $p+1$. Pour chaque $\alpha$, soit $\mu_{\alpha}$ un homomorphisme de $E_{\alpha}$ sur $E$ tel que $\mu_{\alpha} \lambda_{\alpha}=p \delta_{E}$ et $\lambda_{\alpha} \mu_{\alpha}=p \delta_{E_{\alpha}}$. Nous nous servirons des mêmes notations que dans 6-8. 
Soit $k_{1}$ une extension de degré fini de $K_{p N}{ }^{*}(E)$ telle que tous les $\lambda_{\nu}, \mu_{\nu}$ soient définis par rapport à $k_{1}$. Soit $\mathfrak{p}_{1}$ un diviseur premier de $k_{1}$ prolongeant le diviseur premier $p$ de $\boldsymbol{Q}$ tel que $j\left(\mathfrak{p}_{1}\right)$ soit transcendant sur le corps premier. D'après un résultat classique ${ }^{4)}$, tous les $j_{\alpha}$ sont $\mathfrak{p}_{1}$-entiers. Il existe donc pour chaque $\alpha$ une courbe elliptique $E_{\alpha}{ }^{\prime}$ isomorphe à $E_{\alpha}$ qui n'a pas de défaut pour tout prolongement de $\mathfrak{p}_{1}$. Soient $\lambda_{\alpha^{\prime}}$ un homomorphisme de $E$ sur $E_{\alpha}{ }^{\prime}$ tel que $\mathrm{g}\left(\lambda_{\alpha}{ }^{\prime}\right)=\mathfrak{g}_{\alpha}$ et $\mu_{\alpha}{ }^{\prime}$ un homomorphisme de $E_{\alpha^{\prime}}{ }^{\prime}$ sur $E$ tel que $\mu_{\alpha}{ }^{\prime} \lambda_{\alpha}{ }^{\prime}=p \delta_{E}, \lambda_{\alpha}{ }^{\prime} \mu_{\alpha}{ }^{\prime}=p \delta_{E_{\alpha^{\prime}}}$. Soient $k$ une extension finie de $k_{1}$ par rapport à laquelle tous les $E_{\alpha}{ }^{\prime}$ et tous les $\lambda_{\alpha}{ }^{\prime}, \mu_{\alpha}{ }^{\prime}$ sont définis et $\mathfrak{p}$ un prolongement de $\mathfrak{p}_{1}$ dans $k$. En réduisant modulo $\mathfrak{p}$, on a $\tilde{\mu}_{\alpha}{ }^{\prime} \tilde{\lambda}_{a}{ }^{\prime}=p \delta_{E}^{\tilde{E}}$. D'après la prop. 3 , on a $\nu_{s}\left(\tilde{\mu}_{\alpha}^{\prime}\right) \nu_{s}\left(\tilde{\lambda}_{\alpha}^{\prime}\right)=p$; il en résulte que l'on a $\nu_{s}\left(\tilde{\mu}_{\alpha}^{\prime}\right)=1$ ou $\nu_{s}\left(\tilde{\lambda}_{\alpha}^{\prime}\right)=1$. D'après la prop. 2 , on a $\tilde{j}_{\alpha}=\hat{j}^{p}$ ou $\tilde{j}_{\alpha}{ }^{p}=\tilde{j}$; par suite tous les $\tilde{j}_{\alpha}$ sont transcendants sur le corps premier. Comme $E_{\alpha}$ est défini par l'équation (6) et $\gamma_{\alpha}$ $=27 j_{\alpha} /\left(j_{\alpha}-1\right), E_{\alpha}$ n'a pas de défaut pour $\mathfrak{p} ; \tilde{E}_{\alpha}$ est alors défini par l'équation

$$
Y^{2}=4 X^{3}-\tilde{\gamma}_{\alpha} X-\tilde{\gamma}_{\alpha} .
$$

La réduction modulo $\mathfrak{p}$ donne un homomorphisme de $\mathfrak{g}(p, E) \operatorname{sur} \mathfrak{g}(p, \tilde{E})$. Comme le groupe $\mathrm{g}(p, E)$ est d'ordre $p^{2}$ et comme, d'après la prop. 3 , le groupe $g(p, \widetilde{E})$ est d'ordre $p$, le noyau de cet homomorphisme est d'ordre $p$; donc il coïncide avec un des $\mathfrak{g}_{\alpha}$, mettons $\mathfrak{g}_{1}$. On a alors $\mathfrak{g}\left(\tilde{\lambda}_{1}\right)=\tilde{\mathfrak{g}}_{1}=\{0\}$. Si $\alpha>1$, on a $\mathfrak{g}(p, E)=\mathfrak{g}_{1}+\mathfrak{g}_{\alpha}$, de sorte qu'on a $\mathfrak{g}\left(\tilde{\lambda}_{\alpha}\right)=\tilde{\mathfrak{g}}_{\alpha}=\mathfrak{g}(p, \tilde{E})$. On a donc $\nu_{s}\left(\tilde{\lambda}_{1}\right)=1$ et $\nu_{s}\left(\tilde{\lambda}_{\alpha}\right)=p$ pour $\alpha>1$. Comme on a $\tilde{\mu}_{\alpha} \tilde{\lambda}_{\alpha}=p \delta_{\tilde{E}}$, d'après la prop. 3 on a $\nu_{s}\left(\tilde{\mu}_{1}\right)=p$ et $\nu_{s}\left(\tilde{\mu}_{\alpha}\right)=1$ pour $\alpha>1$. Il en résulte d'après la prop. 2, qu'il y a un isomorphisme $\varepsilon$ de $\tilde{E}^{p}$ sur $\tilde{E}_{1}$ et un isomorphisme $\varepsilon_{\alpha}$ de $\widetilde{E}_{\alpha}^{p}$ sur $\tilde{E}$ pour chaque $\alpha>1$ tels que

On a donc

$$
\begin{aligned}
& \tilde{\lambda}_{1} \tilde{x}=\varepsilon \tilde{x}^{p} \quad \text { pour } \quad \tilde{x} \in \tilde{E}, \\
& \tilde{\mu}_{\alpha} \tilde{x}_{\alpha}=\varepsilon_{\alpha} \tilde{x}_{\alpha}^{p} \quad \text { pour } \quad \tilde{x}_{\alpha} \in \tilde{E}_{\alpha} \quad(\alpha>1) .
\end{aligned}
$$

$$
\begin{array}{ll}
\tilde{j}_{1}=\tilde{\jmath}^{p}, & \tilde{\gamma}_{1}=\tilde{\gamma}^{p}, \\
\tilde{j}=\tilde{\jmath}_{\alpha}{ }^{p}, & \tilde{\gamma}=\tilde{\gamma}_{\alpha}{ }^{p} \quad(\alpha>1) .
\end{array}
$$

On en déduit que $\tilde{E}_{1}=\tilde{E}^{p}, \tilde{E}=\tilde{E}_{\alpha}^{p}(\alpha>1)$ comme les équations de $E$ et de $E_{\alpha}$ ont les formes (4) et (6); les isomorphismes $\varepsilon, \varepsilon_{\alpha}$ sont donc éguax à \pm 1 . Soient $h$ et $h_{\alpha}$ respectivement les fonctions canoniques sur $E$ et sur $E_{\alpha}$ comme dans $6 \sim 8$; on a alors $\tilde{h}_{1}=\tilde{h}^{p}$ et $\tilde{h}=\tilde{h}_{\alpha}{ }^{p}$ pour $\alpha>1$. Il s'ensuit donc de la relation (13) que

$$
\begin{array}{lll}
\tilde{h}_{1}\left(\tilde{\lambda}_{1} \tilde{t}\right)=\tilde{h}^{p}\left( \pm \tilde{t}^{p}\right)=\tilde{h}(\tilde{t})^{p} & \text { pour } \quad \tilde{t} \in \mathfrak{g}(N, \tilde{E}), & \\
\tilde{h}\left(\tilde{\mu}_{\alpha} \tilde{t}_{\alpha}\right)=\tilde{h}_{\alpha}^{p}\left( \pm \tilde{t}_{\alpha}^{p}\right)=\tilde{h}_{\alpha}\left(\tilde{t}_{\alpha}\right)^{p} & \text { pour } \quad \tilde{t}_{\alpha} \in \mathfrak{g}\left(N, \tilde{E}_{\alpha}\right), \quad(\alpha>1) .
\end{array}
$$

4) Une démonstration moderne de ce résultat est donnée dans [2] $\S 6$. 
En substituant $\tilde{\lambda}_{\alpha} \tilde{t}$ à $\tilde{t}_{\alpha}$ on obtient

$$
\tilde{h}_{\alpha}\left(\tilde{\lambda}_{\alpha} \tilde{t}\right)^{p}=\tilde{h}(p \tilde{t}) \quad \text { pour } \quad \tilde{t} \in g(N, \tilde{E}) .
$$

Soient $\sigma_{\alpha}$ pour $1 \leqq \alpha \leqq p+1$ et $\rho_{n}$ les isomorphismes du corps $K_{N}(E)$ définis dans 6. Les relations (14), (15), (15') montrent que $K_{N}$ a un système de générateurs $(x)=\left(x_{1}, \cdots, x_{c}\right)$ sur $\boldsymbol{Q}$ tel que tous les $x_{i}, x_{i}{ }^{\sigma_{\alpha}}, x_{i}{ }^{\rho p}$ soient $\mathfrak{p}$-entiers et que l'on ait

$$
x_{i}^{\sigma_{1}} \equiv x_{i}^{p} \quad \text { mod. } \mathfrak{p}
$$

$$
\left(x_{i}{ }^{\sigma}\right)^{p} \equiv x_{i}{ }^{{ }^{p} p}(1<\alpha \leqq p+1) \quad \text { mod. } \mathfrak{p} .
$$

11. Nous allons maintenant nous occuper des correspondances définies dans 7. Soient $L$ un sous-corps de $K_{N}$ tel que $L \supset \boldsymbol{Q}(j), L \cap \boldsymbol{Q}\left(\zeta_{N}\right)=\boldsymbol{Q}$ et $\{\Gamma, u\}$ un modèle de $L$. Soient $X_{p}, Y_{p}$ les correspondances sur $\Gamma$ définies dans 7 . $\Gamma$ n'a pas de défaut pour presque tous les diviseurs premiers de $\boldsymbol{Q}$. Pour chacun de ces diviseurs premiers de $\boldsymbol{Q}$, nous prenons un de ses prolongements dans $\boldsymbol{F}$ et le fixons; nous désignons le diviseur premier de $\boldsymbol{F}$ prolongeant ( $p$ ) aussi par $p$. On vérifie facilement que le point $u$ reste générique par rapport au corps premier modulo presque tous les $p$.

Soient $u_{1}, \cdots, u_{z}$ les coordonnées du point $u$; chaque $u_{i}$ s'exprime sous la forme $u_{i}=f_{i}(x) / g_{i}(x)$ où $f_{i}, g_{i}$ sont deux polynomes à coefficients entiers. Tous les $g_{i}(x), g_{i}(x)^{\rho_{n}}(1 \leqq i \leqq z, 1 \leqq n \leqq N,(n, N)=1)$ sont des $p$-unités pour presque tous les diviseurs premiers $p$. La formule (16) montre que les $g_{i}(x)^{\sigma_{\alpha}}$ sont des $p$-unités pour ces $p$. Il s'ensuit donc de (16) que l'on a pour presque tous les $p$,

$$
\tilde{u}^{\sigma_{1}}=\tilde{u}^{p}, \quad\left(\tilde{u}^{\sigma_{\alpha}}\right)^{p}=\tilde{u}^{\rho p} \quad(\alpha>1) .
$$

Si $\tilde{u}$ est générique sur $\tilde{\Gamma}$ par rapport au corps premier, les produits d'intersection $\tilde{X}_{p} \cdot(\tilde{u} \times \tilde{\Gamma})$ et $\tilde{Y}_{p} \cdot(\tilde{u} \times \tilde{\Gamma})$ sont définis. Il résulte alors de (7), (9), (17) et du th. 17 de [5] que l'on a

$$
\tilde{X}_{p}(\tilde{u})=\tilde{u}^{p}+p \tilde{Y}_{p}(\tilde{u})^{1 / p} .
$$

$\tilde{Y}_{n}$ donne, pour chaque $n$, une correspondance birationnelle de $\tilde{\Gamma}$ pour presque tous les $p$. Soit $\Pi$ le lieu de $\tilde{u} \times \tilde{u}^{p}$ sur $\tilde{\Gamma} \times \tilde{\Gamma}$ par rapport au corps premier. Alors on a

$$
\tilde{X}_{p}(\tilde{u})=\left(\Pi+\Pi^{\prime} \circ \tilde{Y}_{p}\right)(\tilde{u}) ;
$$

on en déduit que $\tilde{X}_{p}-\left(\Pi+\Pi^{\prime} \circ \tilde{Y}_{p}\right)$ est un cycle $a \times \tilde{\Gamma}$ où a est un diviseur sur $\tilde{\Gamma}$ en vertu du th. 2 de [10]. Comme $\Pi+\Pi^{\prime} \circ \tilde{Y}_{p}$ n'a pas de composant de la forme $a \times \tilde{\Gamma}$, on voit que $a>0$. D'autre part on a $d\left(\tilde{X}_{p}\right)=d^{\prime}\left(\tilde{X}_{p}\right)=p+1$ en vertu de $(8)$ et $d^{\prime}\left(\Pi+\Pi^{\prime} \circ \tilde{Y}_{p}\right)=p+1$; d'où résulte $\mathfrak{a}=0$. Nous obtenons ainsi la première formule de congruence pour la correspondance modulaire 


$$
\tilde{X}_{p}=\Pi+\Pi^{\prime} \circ \tilde{Y}_{p}
$$

pour presque tous les nombres premiers $p$.

12. Nous prenons maintenant pour $L$ le corps $L_{N}$ ou le corps $M_{N, \text { h }}$ définis dans 8. Soient les notations $A, B, \varphi_{n}$ comme dans 8. D’après la prop. 7, $\varphi_{p}$ donne un automorphisme de Frobenius $\left(\frac{\boldsymbol{Q}\left(\boldsymbol{\zeta}_{N}\right) / \boldsymbol{Q}}{p}\right)$. Il s'ensuit de là que l'on a $\tilde{A}^{\varphi p}=\tilde{A}^{p}, \tilde{B}^{\varphi p}=\tilde{B}^{p}$. D'après la prop. 12 et la prop. 13 , on a

$$
\begin{aligned}
& \tilde{A}^{p} \circ \tilde{Y}_{p}=\tilde{A} \quad \text { sur } \tilde{\Gamma}_{N}, \\
& \tilde{B}^{p} \circ \tilde{Y}_{p}=\tilde{B} \quad \text { sur } \tilde{\Gamma}_{N, \mathfrak{h}} .
\end{aligned}
$$

Comme $A$ est une correspondance birationnelle, on a $A^{\prime} \circ A=\Delta$ et par conséquent

$$
\tilde{A}^{\prime} \circ \tilde{A}=\tilde{\Delta} .
$$

D’autre part, on vérifie aisément

$$
\Pi \circ \mathfrak{X}=\mathfrak{X}^{p} \circ \Pi \text { et } \mathfrak{X} \circ \Pi^{\prime}=\Pi^{\prime} \circ \mathfrak{X}^{p}
$$

pour toute correspondance $\mathfrak{X}$. Il s'ensuit alors des formules (18), (19), (20) que l'on a

$$
\tilde{A}^{\prime} \circ \Pi^{\prime} \circ \tilde{A}=\tilde{A}^{\prime} \circ \Pi^{\prime} \circ \tilde{A}^{p} \circ \tilde{Y}_{p}=\tilde{A}^{\prime} \circ \tilde{A} \circ \Pi^{\prime} \circ \tilde{Y}_{p}=\Pi^{\prime} \circ \tilde{Y}_{p}
$$

sur $\tilde{\Gamma}_{N}$. Il en est de même pour $\tilde{B}, \tilde{\Gamma}_{N, \mathfrak{l}}$. On obtient ainsi la deuxième formule de congruence:

$$
\begin{aligned}
& \Pi^{\prime} \circ \tilde{Y}_{p}=\tilde{A}^{\prime} \circ \Pi^{\prime} \circ \tilde{A} \text { sur } \tilde{\Gamma}_{N}, \\
& \Pi^{\prime} \circ \tilde{Y}_{p}=\tilde{B}^{\prime} \circ \Pi^{\prime} \circ \tilde{B} \text { sur } \tilde{\Gamma}_{N, \mathfrak{h}}
\end{aligned}
$$

pour presque tous les nombres premiers $p$.

13. Nous allons récrire les formules (I), (II) en termes d'endomorphismes de la jacobienne.

Soient $L, \Gamma$ comme dans 11, $J$ une jacobienne de $\Gamma$ et $\varphi$ une application canonique de $\Gamma$ dans $J$. Comme $\Gamma$ est défini par rapport à $\boldsymbol{Q}$, on peut supposer d'après [1] ou d'après [12] que $J$ soit défini par rapport à $\boldsymbol{Q}$; de plus si $\Gamma$ a un point rationnel par rapport à $\boldsymbol{Q}$, on peut aussi supposer que $\varphi$ soit défini par rapport à $\boldsymbol{Q}$. Cela posé, nous désignerons par $\xi_{n}$ et $\eta_{n}$ les endomorphismes de $J$ correspondant à $X_{n}$ et à $Y_{n}$. Nous désignerons la jacobienne $J$ de $\Gamma$ par $J_{N}$ ou $J_{N, \text { b }}$ selon que $L$ est égal à $L_{N}$ ou à $M_{N, \text { b }}$; nous désignerons aussi par $\alpha$ et $\beta$ les endomorphismes de $J_{N}$ et de $J_{N, \hbar}$ correspondant à $A$ et à $B$, respectivement. Nous démontrerons, dans 17 , que $\Gamma_{N}, \Gamma_{N, \mathfrak{G}}$ ont des points rationnels par rapport à $\boldsymbol{Q}$. 
Pour presque tous les nombres premiers $p, J$ n'a pas de défaut, $\tilde{J}$ est une jacobienne de $\tilde{\Gamma}$ et $\tilde{\varphi}$ est une application canonique dans $\tilde{\Gamma}$. D'après (I), (II), nous obtenons donc les relations suivantes pour presque tous les nombres premiers $p$ :

$$
\begin{array}{ll}
\tilde{\xi}_{p}=\pi+\pi^{\prime} \tilde{\eta}_{p} & \text { sur } J, \\
\pi^{\prime} \tilde{\eta}_{p}=\tilde{\alpha}^{-1} \pi^{\prime} \tilde{\alpha} & \text { sur } J_{N}, \\
\pi^{\prime} \tilde{\eta}_{p}=\tilde{\beta}^{-1} \pi^{\prime} \tilde{\beta} & \text { sur } J_{N, \boldsymbol{\eta}}
\end{array}
$$

où $\pi$ désigne l'homomorphisme de $p$-ième puissance de $J$.

\section{$\S 4$. Fonctions modulaires elliptiques.}

14. Soient $\omega_{1}, \omega_{2}$ deux nombres complexes tels que $\Im\left(\omega_{1} / \omega_{2}\right)>0$ et $D$ le réseau dans le plan complexe $\boldsymbol{C}$ engendré par $\left\{\omega_{1}, \omega_{2}\right\}$. Posons

$$
\begin{aligned}
& g_{2}(D)=g_{2}\left(\omega_{1}, \omega_{2}\right)=60 \Sigma^{\prime} \omega^{-4}, \\
& g_{3}(D)=g_{3}\left(\omega_{1}, \omega_{2}\right)=140 \Sigma^{\prime} \omega^{-6}, \\
& \gamma(z ; D)=\gamma\left(z ; \omega_{1}, \omega_{2}\right)=z^{-2}+\Sigma^{\prime}\left[(z-\omega)^{-2}-\omega^{-2}\right]
\end{aligned}
$$

où $\Sigma^{\prime}$ désigne la sommation étendue à tous les nombres $\omega \neq 0$ de $D$. La fonction $\gamma$ et sa dérivée $\gamma^{\prime}$ satisfont à l'équation

$$
\gamma^{\prime 2}=4 \wp^{3}-g_{2} 反-g_{3} .
$$

$g_{2}\left(\omega_{1}, \omega_{2}\right)$ et $g_{3}\left(\omega_{1}, \omega_{2}\right)$ sont les formes modulaires d'espèce ("Stufe») 1 respectivement de degré -4 et -6 . Elles ont les expressions

$$
\begin{aligned}
& g_{2}\left(\omega_{1}, \omega_{2}\right)=\left(\frac{\pi}{\omega_{2}}\right)^{4}\left(\frac{1}{12}+20 \sum_{n=1}^{\infty} \frac{n^{3} q^{n}}{1-q^{n}}\right), \\
& g_{3}\left(\omega_{1}, \omega_{2}\right)=\left(\frac{\pi}{\omega_{2}}\right)^{6}\left(\frac{1}{216}-\frac{7}{3} \sum_{n=1}^{\infty} \frac{n^{5} q^{n}}{1-q^{n}}\right) .
\end{aligned}
$$

où $q=e^{2 \pi i \tau}, \tau=\omega_{1} / \omega_{2}$. Nous nous servirons des notations $q, \tau$ toujours en ce sens et posons aussi $q_{N}=e^{2 \pi i \tau / N}, \zeta_{N}=e^{2 \pi i / N}$.

La fonction $\boldsymbol{j}(\tau)=g_{2}{ }^{3} /\left(g_{2}{ }^{3}-27 g_{3}{ }^{2}\right)$ est une fonction modulaire d'espèce 1 ; elle a l'expression

$$
\boldsymbol{j}(\tau)=12^{-3}\left(q^{-1}+744+\cdots\right)
$$

dont les coefficients sont rationnels. Soient $N$ un entier $>1$ et $\alpha, \beta$ deux entiers tels que $(\alpha, \beta) \neq(0,0)$ mod. $N$. La fonction

$$
\gamma\left(\frac{\alpha \omega_{1}+\beta \omega_{2}}{N} ; \omega_{1}, \omega_{2}\right)
$$

est une forme modulaire d'espèce $N$ et de degré -2 ; elle a l'expression 


$$
\begin{gathered}
\delta\left(\frac{\alpha \omega_{1}+\beta \omega_{2}}{N} ; \omega_{1}, \omega_{2}\right)=\left(\frac{2 \pi}{\omega_{2}}\right)^{2}\left\{-\frac{1}{12}+2 \sum_{n=1}^{\infty} \frac{n q^{n}}{1-q^{n}}-\frac{\zeta_{N}{ }^{\beta} q_{N}{ }^{\alpha}}{\left(1-\zeta_{N}{ }^{\beta} q_{N}{ }^{\alpha}\right)^{2}}\right. \\
\left.-\sum_{n=1}^{\infty} \frac{n q^{n}}{1-q^{n}}\left(\zeta_{N}{ }^{n} q_{N}{ }^{n \alpha}+\zeta_{N}{ }^{n} \beta q_{N}{ }^{n \alpha}\right)\right\} \\
(0 \leqq \alpha<N,(\alpha, \beta) \neq(0,0) \text { mod. } N) .
\end{gathered}
$$

Posons maintenant

$$
\boldsymbol{f}_{\alpha \beta}(\tau)=\boldsymbol{f}(\alpha, \beta ; \tau)=\frac{g_{2}\left(\omega_{1}, \omega_{2}\right)}{g_{3}\left(\omega_{1}, \omega_{2}\right)} \gamma\left(\frac{\alpha \omega_{1}+\beta \omega_{2}}{N} ; \omega_{1}, \omega_{2}\right) .
$$

Les fonctions $\boldsymbol{f}_{\alpha \beta}(\tau)$ sont des fonctions modulaires d'espèce $N$. Pour qu'on ait $\boldsymbol{f}_{\alpha \beta}=\boldsymbol{f}_{\alpha^{\prime} \beta^{\prime}}$, il faut et il suffit que $(\alpha, \beta) \equiv \pm\left(\alpha^{\prime}, \beta^{\prime}\right) \bmod . N$. Soient $a, b, c, d$ quatre entiers rationnels tels que $a d-b c=1$; on vérifie alors facilement que

$$
\boldsymbol{f}\left(\alpha, \beta ; \frac{a \tau+b}{c \tau+d}\right)=\boldsymbol{f}\left(\alpha^{\prime}, \beta^{\prime} ; \tau\right) \text { pour }\left(\alpha^{\prime} \beta^{\prime}\right)=(\alpha \quad \beta)\left(\begin{array}{ll}
a & b \\
c & d
\end{array}\right) \text {. }
$$

D'après (21), (23), on voit que tous les $\boldsymbol{f}_{\alpha \beta}(\tau)$ ont des développements en $q_{N}$ à coefficients dans $\boldsymbol{Q}\left(\zeta_{N}\right)$ et qu'en particulier, $\boldsymbol{f}_{10}(\tau)$ a un développement en $q_{N}$ à coefficients rationnels; si $N \geqq 3, \boldsymbol{f}_{1 \beta}(\tau)$ a un développement en $q_{N}$

$$
\boldsymbol{f}_{1 \beta}(\tau)=r_{\beta}+s_{\beta} \zeta_{N}{ }^{\beta} q_{N}+\text { (termes d'ordres plus hauts) }
$$

où $r_{\beta}, s_{\beta}$ sont deux nombres rationnels et $s_{\beta} \neq 0$.

15. Soit maintenant $\tau_{0}$ un nombre complexe tel que $\boldsymbol{j}\left(\tau_{0}\right)$ soit transcendant sur $\boldsymbol{Q}$. Posons $j_{0}=\boldsymbol{j}\left(\tau_{0}\right)$ et $\gamma_{0}=27 j_{0} /\left(j_{0}-1\right)$; soient $E$ la courbe elliptique définie par l'equation $Y^{2}=4 X^{3}-\gamma_{0} X-\gamma_{0}$ et $h$ la fonction canonique sur $E$. Soient $\omega_{01}, \omega_{02}$ deux nombres complexes tels que $\omega_{01} / \omega_{02}=\tau_{0}$ et $D$ le réseau engendré par $\left\{\omega_{01}, \omega_{02}\right\}$. Il existe alors un isomorphisme analytique $u(z)$ de $\boldsymbol{C} / D$ sur $E$. Nous allons démontrer que l'on a

$$
h(u(z))=\frac{g_{2}\left(\omega_{01}, \omega_{02}\right)}{g_{3}\left(\omega_{01}, \omega_{02}\right)} \gamma\left(z ; \omega_{01}, \omega_{02}\right)
$$

pour tout $z \in \boldsymbol{C}$. Posons $\mu=\left[g_{3}(D) / g_{2}(D)\right]^{1 / 2}$; on a alors $g_{2}(\mu D)=g_{3}(\mu D)$. Puisqu'on a $j_{0}=\boldsymbol{j}\left(\tau_{0}\right)=g_{2}(\mu D) /\left[g_{2}(\mu D)-27 g_{2}(\mu D)\right]$, on voit que $\gamma_{0}=g_{2}(\mu D)=g_{3}(\mu D)$. Par suite, pour tout nombre complexe $z, u^{\prime}(z)=\left(\gamma(\mu z ; \mu D), \gamma^{\prime}(\mu z ; \mu D)\right)$ est un point sur $E$; de plus $z \rightarrow u^{\prime}(z)$ donne un isomorphisme analytique de $\boldsymbol{C} / D$ sur $E$. On a $h\left(u^{\prime}(z)\right)=\gamma(\mu z ; \mu D)=\mu^{-2} \gamma(z ; D)=\frac{g_{2}(D)}{g_{3}(D)} \gamma(z ; D)$. Comme $\mathscr{A}(E)$ est isomorphe à $\boldsymbol{Z}$, on a $u(z)= \pm u^{\prime}(z)$; ce qui montre (26). Posons $t_{1}=u\left(\omega_{01} / N\right), t_{2}=u\left(\omega_{02} / N\right) ;\left\{t_{1}, t_{2}\right\}$ est alors un système de générateurs pour $\mathfrak{g}(N, E)$. D'après (26), on vérifie aisément

$$
\begin{gathered}
j(E)=\boldsymbol{j}\left(\tau_{0}\right), \\
h\left(\alpha t_{1}+\beta t_{2}\right)=\boldsymbol{f}_{\alpha \beta}\left(\tau_{0}\right) ;
\end{gathered}
$$


on a donc

$$
K_{N}(E)=\boldsymbol{Q}\left(\boldsymbol{j}\left(\tau_{0}\right), \boldsymbol{f}_{\alpha \beta}\left(\tau_{0}\right) \mid 0 \leqq \alpha<N, 0 \leqq \beta<N,(\alpha, \beta) \neq(0,0) \bmod . N\right) .
$$

Soit $\mathfrak{g}_{(i)}$ le sous-groupe de $E$ engendré par $t_{i}$ pour $i=1,2$. Soient $D_{(1)}$, $D_{(2)}$ les réseaux respectivement engendrés par $\left\{\omega_{01} / N, \omega_{02}\right\}$ et $\left\{\omega_{01}, \omega_{02} / N\right\}$. Alors l'application identique $z \rightarrow z$ sur le plan complexe $\boldsymbol{C}$ donne un homomorphisme de $\boldsymbol{C} / D$ sur $\boldsymbol{C} / D_{(i)}$ dont le noyau est $D / D_{(i)}$ pour $i=1,2$. On peut en déduire que

$$
j\left(E / \mathfrak{g}_{(1)}\right)=\boldsymbol{j}\left(\tau_{0} / N\right), \quad j\left(E / \mathfrak{g}_{(2)}\right)=\boldsymbol{j}\left(N \tau_{0}\right) .
$$

16. Nous désignerons par $\mathfrak{M}_{N}$ le corps complet des fonctions modulaires elliptiques d'espèce $N$. $\mathfrak{M}_{N}$ est un corps de fonctions algébriques d'une variable sur $\boldsymbol{C}$. $\mathfrak{M}_{N}$ est galoisien sur $\mathfrak{M}_{1}=\boldsymbol{C}(\boldsymbol{j}(\tau))$; le groupe de Galois de $\mathfrak{M}_{N} / \mathfrak{M}_{1}$ est isomorphe au groupe $S_{N}$ de telle façon que pour chaque $\left(\begin{array}{ll}a & b \\ c & d\end{array}\right)$ $\in S_{N}^{*}$, l'application

$$
\boldsymbol{f}(\tau) \rightarrow \boldsymbol{f}\left(\frac{a \tau+b}{c \tau+d}\right)
$$

donne un automorphisme de $\mathfrak{M}_{N} / \mathfrak{M}_{1}$.

Nous désignerons par $\Re_{N}$ le corps $\boldsymbol{Q}\left(\boldsymbol{j}(\tau), \boldsymbol{f}_{\alpha \beta}(\tau)\right)$ engendré sur $\boldsymbol{Q}$ par $\boldsymbol{j}(\tau)$ et tous les $\boldsymbol{f}_{\alpha \beta}(\tau)(0 \leqq \alpha \leqq N-1,0 \leqq \beta \leqq N-1,(\alpha, \beta) \neq(0,0)$ mod. $N)$. D’après la relation (24), $a, b, c, d$, ayant les mêmes significations que dans cette relationlà, pour qu'on ait $\boldsymbol{f}\left(\frac{a \tau+b}{c \tau+d}\right)=\boldsymbol{f}(\tau)$ pour tous les $\boldsymbol{f} \in \mathfrak{\Omega}_{N}$, il faut et il suffit qu'on ait $\left(\begin{array}{ll}a & b \\ c & d\end{array}\right) \equiv \pm I$ mod. $N$. Cela démontre que $\mathfrak{R}_{N}$ engendre $\mathfrak{M}_{N}$ sur $\boldsymbol{C}$, c'est-à-dire

$$
\mathfrak{M}_{N}=\boldsymbol{C}\left(\boldsymbol{j}(\tau), \boldsymbol{f}_{\alpha \beta}(\tau)\right)
$$

$E$ étant la courbe elliptique avec l'invariant $j$ transcendant sur $\boldsymbol{Q}$, définie dans 4 , nous allons démontrer que $K_{N}(E)$ est isomorphe au corps $\Re_{N}$. Soit $k$ le corps de constantes du corps $\mathscr{R}_{N} ; \mathfrak{\Re}_{N}$ est alors un corps de fonctions algébrique d'une variable sur $k$. Comme $\mathfrak{R}_{N}$ est engendré par l'adjonction à $\boldsymbol{Q}$ de quantités en nombre fini, il en est de même pour $k$; on peut donc prendre un nombre complexe $\tau_{0}$ tel que $\boldsymbol{j}\left(\tau_{0}\right)$ soit transcendant sur $k$. On voit que $\left(\boldsymbol{j}\left(\tau_{0}\right), \boldsymbol{f}_{\alpha \beta}\left(\tau_{0}\right)\right)$ est une spécialisation de $\left(\boldsymbol{j}(\tau), \boldsymbol{f}_{\alpha \beta}(\tau)\right)$ par rapport à $\boldsymbol{C}$, et donc naturellement par rapport à $k$. D’après la définition de $k$, $\left(\boldsymbol{j}(\tau), \boldsymbol{f}_{\alpha \beta}(\tau)\right)$ est de dimension 1 sur $k$. Comme $\boldsymbol{j}\left(\tau_{0}\right)$ est transcendant sur $k$, $\left(\boldsymbol{j}\left(\tau_{0}\right), \boldsymbol{f}_{\alpha \beta}\left(\tau_{0}\right)\right)$ est une spécialisation générique de $\left(\boldsymbol{j}(\tau), \boldsymbol{f}_{\alpha \beta}(\tau)\right)$ par rapport à $k$ et donc par rapport à $\boldsymbol{Q}$, de sorte que $\mathscr{R}_{N}=\boldsymbol{Q}\left(\boldsymbol{j}(\tau), \boldsymbol{f}_{\alpha_{\beta}}(\tau)\right)$ est isomorphe à $\boldsymbol{Q}\left(\boldsymbol{j}\left(\tau_{0}\right), \boldsymbol{f}_{\alpha \beta}\left(\tau_{0}\right)\right)$. Nous avons vu dans 15, que l'on a $\boldsymbol{Q}\left(\boldsymbol{j}\left(\tau_{0}\right), \boldsymbol{f}_{\alpha \beta}\left(\tau_{0}\right)\right)=K_{N}\left(E_{0}\right)$ pour une courbe elliptique $E_{0}$ définie par l'équation

$$
Y^{2}=4 X^{3}-r_{0} X-r_{0}
$$


où $\gamma_{0}=27 \boldsymbol{j}\left(\tau_{0}\right) /\left[\boldsymbol{j}\left(\tau_{0}\right)-1\right] . \quad \mathfrak{R}_{N}$ est donc isomorphe à $K_{N}\left(E_{0}\right)$; de plus on voit que $\left(\boldsymbol{j}(\tau), \boldsymbol{f}_{\alpha \beta}(\tau)\right)$ est de dimension 1 sur $\boldsymbol{Q}$. L'argument ci-dessus est valable pour $E_{0}$ si seulement $\boldsymbol{j}\left(\tau_{0}\right)$ est transcendant sur $\boldsymbol{Q}$. Par suite on peut exprimer les propositions sur $\mathfrak{\Re}_{N}$ en termes de courbe elliptique, au moyen de l'isomorphisme donné ainsi par une spécialisation $\tau \rightarrow \tau_{0}$ où $\tau_{0}$ est un nombre complexe tel que $\boldsymbol{j}\left(\tau_{0}\right)$ soit transcendant sur $\boldsymbol{Q}$. Nous appellerons cette méthode le principe de spécialisation.

17. Nous allons maintenant démontrer les propositions 5-7. Pour cela nous démontrons d'abord le lemme suivant:

Lemme 4. Soient $k_{0}$ un corps, $k_{1}$ une extension séparable de $k_{0}, x$ une variable sur $k_{1}$ et $M$ une extension de $k_{0}(x)$, de degré fini, telle que $M \subset k_{1}(x)$. Alors il existe une extension $k$ de $k_{0}$, de degré fini, telle que $M=k(x)$.

Soit $\bar{k}_{0}$ la clôture algébrique de $k_{0}$. Comme $\left[M: k_{0}(x)\right]$ est fini, il existe un ensemble fini $(y)=\left(y_{1}, \cdots, y_{s}\right)$ d'éléments de $k_{1}$ tel que $M \subset k_{0}(x, y)$. Comme $x$ est une variable sur $\bar{k}_{0}(y)$ et comme $\bar{k}_{0}(y)$ est une extension régulière de $\bar{k}_{0}, \bar{k}_{0}(x, y)$ est une extension régulière de $\bar{k}_{0}(x)$; par suite $\bar{k}_{0}(x)$ est algébriquement fermé dans $\bar{k}_{0}(x, y)$; il en résulte que $\bar{k}_{0}(x)=\bar{k}_{0} M$; on a donc $M \subset \bar{k}_{0}(x)$. Il existe donc une extension $k^{\prime}$ de $k_{0}$, de degré fini, telle que $M \subset k^{\prime}(x)$. Posons $k=M \cap k^{\prime}$; on a alors $k(x) \subset M$ et $\bar{k} \cap M=\bar{k} \cap k^{\prime}(x) \cap M=k^{\prime} \cap M=k$. D'autre part, puisque $k_{1}$ est séparable sur $k_{0}$, on voit que $M$ est une extension séparable de $k$; on en conclut que $M$ est une extension régulière de $k$. On a donc

$$
\left[k^{\prime}(x): M\right]=\left[k^{\prime} M: M\right]=\left[k^{\prime}: k\right]=\left[k^{\prime}(x): k(x)\right] ;
$$

ce qui montre $M=k(x)$.

$K_{N}(E)$ est identifié avec $\mathscr{\Re}_{N}$ par l'isomorphisme donné dans 16; nous fixerons cette identification. On a alors

$$
\begin{array}{ll}
j_{(1)}=\boldsymbol{j}(\tau / N), & j_{(2)}=\boldsymbol{j}(N \tau), \\
h\left(t_{1}\right)=\boldsymbol{f}_{10}(\tau), & h\left(t_{2}\right)=\boldsymbol{f}_{01}(\tau)
\end{array}
$$

en vertu de (27), (28), La formule (5) prend maintenant la forme

$$
\boldsymbol{f}_{\alpha \beta}{ }^{\sigma}=\boldsymbol{f}_{\alpha^{\prime} \beta^{\prime}}, \quad\left(\alpha^{\prime} \beta^{\prime}\right)=\left(\begin{array}{ll}
\alpha & \beta
\end{array}\right)\left(\begin{array}{ll}
a & b \\
c & d
\end{array}\right) \text {; }
$$

cette formule signifie que pour chaque automorphisme $\sigma$ de $\Re_{N}$ sur $\boldsymbol{Q}(\boldsymbol{j}(\tau))$ il existe une telle matrice $\left(\begin{array}{ll}a & b \\ c & d\end{array}\right) \in G_{N}{ }^{*}$. L'application $\sigma \rightarrow\left(\begin{array}{ll}a & b \\ c & d\end{array}\right)$ est un isomorphisme dans $G_{N}=G_{N}{ }^{*} /\{ \pm I\}$. Soit $G_{N}{ }^{\prime}$ l'image de cet isomorphisme. Il est bien connu que chaque élément de $S_{N}{ }^{*}$ est représenté par une matrice $\left(\begin{array}{ll}a & b \\ c & d\end{array}\right)$ à coefficients entiers telle que $a d-b c=1$ (non seulement $a d-b c \equiv 1$ 
mod. $N)$. L'application $\tau \rightarrow \frac{a \tau+b}{c \tau+d}$ donne un automorphisme de $\mathfrak{M}_{N}$ sur $\boldsymbol{Q}(\boldsymbol{j}(\tau))$; par suite on voit que $G_{N}{ }^{\prime} \supset S_{N}$ en vertu de (24), Puisque le groupe quotient $G_{N} / S_{N}$ est isomorphe au groupe $(\boldsymbol{Z} / N \boldsymbol{Z})^{\times}$d'éléments inversibles de $\boldsymbol{Z} / N \boldsymbol{Z}$, il existe un sous-groupe $\mathfrak{h}$ de $(\boldsymbol{Z} / N \boldsymbol{Z})^{\times}$tel qu'on ait

$$
G_{N}{ }^{\prime}=\left\{\left(\begin{array}{ll}
a & b \\
c & d
\end{array}\right) \mid a d-b c \in \mathfrak{h}\right\} /\{ \pm I\} .
$$

Soit $H^{\prime}$ le sous-groupe

$$
\left\{\left(\begin{array}{rr} 
\pm 1 & 0 \\
0 & a
\end{array}\right) \mid(a, N)=1\right\} /\{ \pm I\}
$$

de $G_{N}$. On a alors

$$
\begin{gathered}
G_{N}{ }^{\prime} \cap H^{\prime}=\left\{\left(\begin{array}{rr} 
\pm 1 & 0 \\
0 & a
\end{array}\right) \mid \pm a \in \mathfrak{h}\right\}, \\
\left(G_{N}{ }^{\prime} \cap H^{\prime}\right) S_{N}=G_{N}{ }^{\prime}, \quad\left(G_{N}{ }^{\prime} \cap H^{\prime}\right) \cap S_{N}=\{e\} .
\end{gathered}
$$

Soit $\mathfrak{Z}$ le sous-corps de $\Re_{N}$ correspondant à $S_{N}$; alors on a $\boldsymbol{Q}(\boldsymbol{j}) \subset \mathbb{L} \subset \boldsymbol{C}(\boldsymbol{j})$. Comme $\mathfrak{L}$ est une extension de $\boldsymbol{Q}(\boldsymbol{j})$ de degré fini, il existe, d'après le lemme 4 , une extension $k$ de $\boldsymbol{Q}$ de degré fini telle que $\mathbb{R}=k(\boldsymbol{j})$. Tous les éléments de $\Re_{N} \cap \boldsymbol{C}$ sont fixés par $S_{N}$; on a donc $\mathfrak{R}_{N} \cap \boldsymbol{C} \subset k(\boldsymbol{j})$; d'où résulte $\mathfrak{\Re}_{N} \cap \boldsymbol{C}=k$. Dans 5, nous avons montré, en supposant que $G_{N}=G_{N}{ }^{\prime}$, que $\boldsymbol{Q}\left(j, j_{(2)}, h\left(t_{1}\right)\right)$ correspond à $H^{\prime}$. Quand on ne suppose pas $G_{N}=G_{N}{ }^{\prime}$, on voit que $\boldsymbol{Q}\left(j, j_{(2)}, h\left(t_{1}\right)\right)$ correspond à $H^{\prime} \cap G_{N}{ }^{\prime}$. On a $\boldsymbol{Q}\left(j, j_{2}, h\left(t_{1}\right)\right)=\boldsymbol{Q}\left(\boldsymbol{j}(\tau), \boldsymbol{j}(N \tau), \boldsymbol{f}_{10}(\tau)\right)$ en vertu de notre identification. D'après (30), on a

$$
\begin{gathered}
\boldsymbol{Q}\left(\boldsymbol{j}(\tau), \boldsymbol{j}(N \tau), \boldsymbol{f}_{10}(\tau)\right) \cap k(\boldsymbol{j}(\tau))=\boldsymbol{Q}(\boldsymbol{j}), \\
\mathfrak{\Re}_{N}=k\left(\boldsymbol{j}(\tau), \boldsymbol{j}(N \tau), \boldsymbol{f}_{10}(\tau)\right) .
\end{gathered}
$$

On peut regarder $\Re_{N}$ comme un sous-corps du corps $\boldsymbol{Q}\left(\zeta_{N}\right)\left(\left(q_{N}\right)\right)$ des séries formelles en $q_{N}$ à coefficients dans $\boldsymbol{Q}\left(\zeta_{N}\right)$. L'application $q_{N} \rightarrow 0$ donne un diviseur premier $\mathfrak{P}$ du corps $\mathscr{\Omega}_{N}$. D'après la formule (25), on a

$$
\frac{f_{1 \beta}-r_{\beta}}{f_{1 \beta^{\prime}}-\gamma_{\beta^{\prime}}}(\mathfrak{B})=\frac{s_{\beta}}{s_{\beta^{\prime}}} \zeta_{N^{\beta}}{ }^{\beta^{\prime}} ;
$$

ce qui montre que le corps des restes modulo $\mathfrak{P}$ est $\boldsymbol{Q}\left(\zeta_{N}\right)$. $\mathfrak{P}$ induit un diviseur premier $\mathfrak{p}$ dans $\boldsymbol{Q}\left(\boldsymbol{j}(\tau), \boldsymbol{j}(N \tau), \boldsymbol{f}_{10}(\tau)\right)$. Comme $\boldsymbol{j}(\tau), \boldsymbol{j}(N \tau), \boldsymbol{f}_{10}(\tau)$ ont des développements en $q_{N}$ à coefficients rationnels, le corps des restes modulo $\mathfrak{p}$ est $\boldsymbol{Q}$. On a donc

$$
\left[H^{\prime \prime} \cap G_{N}{ }^{\prime}:\{e\}\right]=\left[\Re_{N}: \boldsymbol{Q}\left(\boldsymbol{j}(\tau), \boldsymbol{j}(N \tau), \boldsymbol{f}_{10}(\tau)\right)\right] \geqq\left[\boldsymbol{Q}\left(\zeta_{N}\right): \boldsymbol{Q}\right]=\left[H^{\prime}:\{e\}\right] ;
$$

d'où résulte $H^{\prime} \cap G_{N}{ }^{\prime}=H^{\prime}$; par suite on a $G_{N}=H^{\prime} S_{N}=G_{N_{N}}{ }^{\prime}$; ce qui démontre la prop. 5.

Puisqu'on a $\mathfrak{R}_{N} \cap \boldsymbol{C}=k$, on a $k \subset \boldsymbol{Q}\left(\zeta_{N}\right)$. D'autre part, on a 


$$
\begin{aligned}
{[k: \boldsymbol{Q}] } & \geqq\left[k\left(j, j_{(2)}, h\left(t_{1}\right)\right): \boldsymbol{Q}\left(j, j_{(2)}, h\left(t_{1}\right)\right)\right] \\
& =\left[\Re_{N}: \boldsymbol{Q}\left(\boldsymbol{j}(\tau), \boldsymbol{j}(N \tau), \boldsymbol{f}_{10}(\tau)\right)\right]=\left[\boldsymbol{Q}\left(\zeta_{N}\right): \boldsymbol{Q}\right] ;
\end{aligned}
$$

il s'ensuit de là que $k=\mathrm{Q}\left(\zeta_{N}\right)$; ce qui prouve la prop. 6. Dans le corps $\boldsymbol{Q}\left(\zeta_{N}\right)\left(\left(q_{N}\right)\right)$, l'application $\zeta_{N} \rightarrow \zeta_{N}{ }^{\alpha}, q_{N} \rightarrow q_{N}$ donne un automorphisme de $\mathfrak{\Re}_{N}$ sur $\boldsymbol{Q}(\boldsymbol{j})$ pour chaque $\alpha$ premier avec $N$. D'après la formule (23), cet automorphisme coïncide avec l'automorphisme correspondant à $\left(\begin{array}{ll}1 & 0 \\ 0 & \alpha\end{array}\right)$. Soit $\left(\begin{array}{ll}a & b \\ c & d\end{array}\right)$ un élément de $G_{N} *$ tel que $a d-b c=\alpha$; alors, puisque chaque élément de $S_{N}$ fixe tous les éléments de $\boldsymbol{Q}\left(\zeta_{N}\right),\left(\begin{array}{ll}a & b \\ c & d\end{array}\right),\left(\begin{array}{ll}1 & 0 \\ 0 & \alpha\end{array}\right)$ donnent le même automorphisme de $\boldsymbol{Q}\left(\zeta_{N}\right)$; ce qui démontre la prop. 7 .

Nous avons vu que $\boldsymbol{Q}\left(j, j_{(2)}, h\left(t_{1}\right)\right)$ a un diviseur premier $\mathfrak{p}$ tel que le corps des restes modulo $\mathfrak{p}$ est $\boldsymbol{Q}$. Cela démontre que $\Gamma_{N}$ a un point rationnel par rapport $\grave{a} \boldsymbol{Q}$ comme $L_{N}=\boldsymbol{Q}\left(j, j_{(1)}, h\left(t_{2}\right)\right)$ est isomorphe à $\boldsymbol{Q}\left(j, j_{(2)}, h\left(t_{1}\right)\right)$. Puisque $M_{N, \text { b }}$ est un sous-corps de $L_{N}$, on voit de même que $\Gamma_{N, \mathfrak{h}}$ a un point rationnel par rapport à $\boldsymbol{Q}$.

18. Nous disons que deux matrices $\left(\begin{array}{ll}a & b \\ c & d\end{array}\right),\left(\begin{array}{ll}a^{\prime} & b^{\prime} \\ c^{\prime} & d^{\prime}\end{array}\right)$ à coefficients entiers sont équivalentes s'il existe une matrice $\left(\begin{array}{ll}\alpha & \beta \\ \gamma & \delta\end{array}\right)$ à coefficients entiers telle que $\alpha \delta-\beta \gamma=1$ et $\left(\begin{array}{ll}a^{\prime} & b^{\prime} \\ c^{\prime} & d^{\prime}\end{array}\right)=\left(\begin{array}{ll}\alpha & \beta \\ \gamma & \delta\end{array}\right)\left(\begin{array}{ll}a & b \\ c & d\end{array}\right)$. Si $p$ est un nombre premier, il y a $p+1$ classes d'équivalence des matrices $\left(\begin{array}{ll}a & b \\ c & d\end{array}\right)$ telles que $a d-b c=p$. Soient $\left(\begin{array}{ll}a_{\nu} & b_{\nu} \\ c_{\nu} & d_{\nu}\end{array}\right)(1 \leqq \nu \leqq p+1)$ les représentants de ces classes; de plus, en supposant que $p$ ne divise pas $N$, on peut les choisir de telle façon que

$$
\left(\begin{array}{ll}
a_{\nu} & b_{\nu} \\
c_{\nu} & d_{\nu}
\end{array}\right) \equiv\left(\begin{array}{ll}
1 & 0 \\
0 & p
\end{array}\right) \quad \text { mod. } N \quad(1 \leqq \nu \leqq p+1) .
$$

Cela posé, soient $\left(\begin{array}{ll}a_{\nu}{ }^{\prime} & b_{\nu}{ }^{\prime} \\ c_{\nu}{ }^{\prime} & d_{\nu}{ }^{\prime}\end{array}\right)$ pour $1 \leqq \nu \leqq p+1$ les matrices telles que

on a alors

$$
\left(\begin{array}{ll}
a_{\nu}^{\prime} & b_{\nu}^{\prime} \\
c_{\nu}^{\prime} & d_{\nu}^{\prime}
\end{array}\right)\left(\begin{array}{ll}
a_{\nu} & b_{\nu} \\
c_{\nu} & d_{\nu}
\end{array}\right)=\left(\begin{array}{cc}
p & 0 \\
0 & p
\end{array}\right)
$$

$$
\left(\begin{array}{ll}
a_{\nu}^{\prime} & b_{\nu}^{\prime} \\
c_{\nu}^{\prime} & d_{\nu}^{\prime}
\end{array}\right) \equiv\left(\begin{array}{ll}
p & 0 \\
0 & 1
\end{array}\right) \quad \text { mod. } N
$$

Soient $\tau_{0}, E, D,\left\{\omega_{01}, \omega_{02}\right\}$ et $u(z)$ comme dans 15 ; posons

$$
\left(\begin{array}{l}
\omega_{\nu 1} \\
\omega_{\nu 2}
\end{array}\right)=p^{-1}\left(\begin{array}{ll}
a_{\nu} & b_{\nu} \\
c_{\nu} & d_{\nu}
\end{array}\right)\left(\begin{array}{l}
\omega_{01} \\
\omega_{02}
\end{array}\right) \quad(1 \leqq \nu \leqq p+1) ;
$$


on a alors

$$
\left(\begin{array}{c}
\omega_{01} \\
\omega_{02}
\end{array}\right)=\left(\begin{array}{ll}
a_{\nu}{ }^{\prime} & b_{\nu}{ }^{\prime} \\
c_{\nu}{ }^{\prime} & d_{\nu}{ }^{\prime}
\end{array}\right)\left(\begin{array}{c}
\omega_{\nu 1} \\
\omega_{\nu 2}
\end{array}\right) \quad(1 \leqq \nu \leqq p+1) .
$$

Soit $D_{\nu}$ le réseau engendré par $\left\{\omega_{\nu 1}, \omega_{\nu 2}\right\}$ pour chaque $\nu$; posons $g_{\nu}=u\left(D_{\nu} / D\right)$. $g_{\nu}$ est un sous-groupe de $E$ d'ordre $p$; on a $g_{\nu} \neq g_{\mu}$ pour $\nu \neq \mu$. Définissons $E_{\nu}, \lambda_{\nu}$ comme dans 6 pour ces $E, \mathfrak{g}_{\nu}$; et posons $u_{\nu}(z)=\lambda_{\nu}(u(z))$; alors $u_{\nu}(z)$ donne un isomorphisme analytique de $C / D_{\nu}$ sur $E_{\nu}$. Soit $h_{\nu}$ la fonction canonique sur $E_{\nu}$. En appliquant la formule (26) à $E_{\nu}$, on a

$$
h_{\nu}\left(u_{\nu}(z)\right)=\frac{g_{2}\left(\omega_{\nu 1}, \omega_{\nu 2}\right)}{g_{3}\left(\omega_{\nu 1}, \omega_{\nu 2}\right)} \gamma\left(z ; \omega_{\nu 1}, \omega_{\nu 2}\right) .
$$

Posons $t_{1}=u\left(\omega_{01} / N\right), t_{2}=u\left(\omega_{02} / N\right)$; on a alors $\alpha t_{1}+\beta t_{2}=u\left(\frac{\alpha \omega_{01}+\beta \omega_{02}}{N}\right)$ et par suite

$$
h_{\nu}\left(\lambda_{\nu}\left(\alpha t_{1}+\beta t_{2}\right)\right)=\frac{g_{2}\left(\omega_{\nu 1}, \omega_{\nu 2}\right)}{g_{3}\left(\omega_{\nu 1}, \omega_{\nu 2}\right)} \gamma\left(\frac{\alpha \omega_{01}+\beta \omega_{02}}{N} ; \omega_{\nu 1}, \omega_{\nu 2}\right) .
$$

D’après les formules (31), (33), on a

$$
\frac{\alpha \omega_{01}+\beta \omega_{02}}{N} \equiv \frac{p \alpha \omega_{\nu 1}+\beta \omega_{\nu 2}}{N} \quad \bmod . D_{\nu}
$$

D’après (32) on a $\omega_{\nu_{1}} / \omega_{\nu 2}=\frac{a_{\nu} \tau_{0}+b_{\nu}}{c_{\nu} \tau_{0}+d_{\nu}}$. Nous obtenons donc

$$
j\left(E_{\nu}\right)=\boldsymbol{j}\left(\frac{a_{\nu} \tau_{0}+b_{\nu}}{c_{\nu} \tau_{0}+d_{\nu}}\right)
$$

$$
h_{\nu}\left(\lambda_{\nu}\left(\alpha t_{1}+\beta t_{2}\right)\right)=\boldsymbol{f}\left(p \alpha, \beta ; \frac{a_{\nu} \tau_{0}+b_{\nu}}{c_{\nu} \tau_{0}+d_{\nu}}\right)
$$

Soient $\tau_{p}^{\prime}$ l'automorphisme de $\mathfrak{R}_{N}$ correspondant à $\left(\begin{array}{ll}p & 0 \\ 0 & 1\end{array}\right)$ et $\bar{\sigma}_{\nu}$ l'isomorphisme de $\mathfrak{\Re}_{N}$ défini par la relation

$$
\boldsymbol{g}^{\bar{\sigma}_{\nu}}(\tau)=\boldsymbol{g}^{\tau} p^{\prime}\left(\frac{a_{\nu} \tau+b_{\nu}}{c_{\nu} \tau+d_{\nu}}\right)
$$

pour $\boldsymbol{g} \in \mathfrak{R}_{N}$. On a alors

$$
\boldsymbol{j}^{\overline{\sigma_{\nu}}}\left(\tau_{0}\right)=j\left(E_{\nu}\right), \quad \boldsymbol{f}_{\alpha \beta}^{\overline{\sigma_{\nu}}}\left(\tau_{0}\right)=h_{\nu}\left(\lambda_{\nu}\left(\alpha t_{1}+\beta t_{2}\right)\right) .
$$

Par le principe de spécialisation, on peut donc identifier $\left(K_{N}, \sigma_{\nu}, K_{N}{ }^{{ }^{\nu}}\right)$ défini dans 6 avec $\left(\Re_{N}, \bar{\sigma}_{\nu}, \Re_{N}^{\bar{\sigma}_{\nu}}\right)$.

Soient $\mathfrak{L}$ un sous-corps de $\mathfrak{R}_{N}$ tel que $\mathfrak{Q} \cap \boldsymbol{Q}\left(\zeta_{N}\right)=\boldsymbol{Q}, \mathfrak{Q} \supset \boldsymbol{Q}(\boldsymbol{j}(\tau))$ et $\{\Gamma, u\}$ un modèle du sous-corps $L$ de $K_{N}$ correspondant à $\mathcal{L}$. Si l'on prend $\boldsymbol{C}$ comme le domaine universel, on peut regarder $\boldsymbol{C} \cdot \Omega$ comme le corps des fonctions sur $\Gamma$, en définissant $\boldsymbol{f}(u)=\boldsymbol{f}\left(\tau_{0}\right)$ pour $\boldsymbol{f} \in \mathfrak{L}$. 
Soit $\mathfrak{L}_{N}$ le sous-corps de $\mathfrak{I}_{N}$ correspondant au sous-groupe $H_{N}$ de $G_{N}$; on a alors

$$
\mathfrak{P}_{N}=\boldsymbol{Q}\left(\boldsymbol{j}(\tau), \boldsymbol{j}(\tau / N), \boldsymbol{f}_{01}(\tau)\right), \quad \mathfrak{M}_{N}=\boldsymbol{C} \mathfrak{Q}_{N} ;
$$

$\mathfrak{L}_{N}$ peut être regardé comme le corps des fonctions sur $\Gamma_{N}$ définies par rapport à $\boldsymbol{Q}$. Puisque $\tau_{p}{ }^{\prime}$ est contenu dans $H_{N}$, on a

$$
\boldsymbol{g}^{\overline{o_{\nu}}}(\tau)=\boldsymbol{g}\left(\frac{a_{\nu} \tau+b_{\nu}}{c_{\nu} \tau+d_{\nu}}\right) \quad(1 \leqq \nu \leqq p+1)
$$

pour $\boldsymbol{g} \in \mathfrak{R}_{N}$; on a donc

$$
\boldsymbol{g}^{\bar{\nu}_{\nu^{\prime}}}(\tau)=\boldsymbol{g}^{\prime}\left(-\frac{a_{\nu} \tau+b_{\nu}}{c_{\nu} \tau+d_{\nu}}\right) \frac{p}{\left(c_{\nu} \tau+d_{\nu}\right)^{2}} \quad(1 \leqq \nu \leqq p+1)
$$

pour $\boldsymbol{g} \in \mathbb{R}_{N}$, où $\boldsymbol{f}^{\prime}(\tau)$ désigne la dérivée de $\boldsymbol{f}(\tau)$.

Soit maintenant $\boldsymbol{f} d \boldsymbol{g}$ une forme différentielle sur $\Gamma_{N}$, de première espèce, où $\boldsymbol{f}, \boldsymbol{g}$ sont deux éléments de $\mathfrak{M}_{N} ; \boldsymbol{f}(\tau) \boldsymbol{g}^{\prime}(\tau)$ est alors une forme parabolique de degré 2, d'espèce $N$. Et réciproquement, chacune de telles formes est obtenue de cette manière. Soit $T_{p}$ l'opérateur de Hecke ([4]); on a alors

$$
\left(\boldsymbol{f} \boldsymbol{g}^{\prime} \mid T_{p}\right)(\tau)=\sum_{\nu=1}^{p+1} \boldsymbol{f}\left(\frac{a_{\nu} \tau+b_{\nu}}{c_{\nu} \tau+d_{\nu}}\right) \boldsymbol{g}^{\prime}\left(\frac{a_{\nu} \tau+b_{\nu}}{c_{\nu} \tau+d_{\nu}}\right) \frac{p}{\left(c_{\nu} \tau+d_{\nu}\right)^{2}} .
$$

Il s'ensuit des formules (35), (36), en vertu d'une proposition de [8], que $\boldsymbol{f g}^{\prime} \mid T_{p}$ correspond à la forme différentielle $\delta X_{p}(\boldsymbol{f} d \boldsymbol{g})$, si $\boldsymbol{f}, \boldsymbol{g}$ sont dans $\mathfrak{L}_{N}$, où $\delta X_{p}$ désigne la différentielle de la correspondance $X_{p}$. En d'autres termes, si l'on désigne par $M^{d}$ une représentation de $\mathcal{A}\left(J_{N}\right)$ par les formes différentielles de première espèce, $M^{d}\left(\xi_{p}\right)$ peut être regardé comme une représentation de $T_{p}$ pour les formes paraboliques de degré 2 , d'espèce $N$.

Soient $\left(\begin{array}{ll}\alpha & \beta \\ \gamma & \delta\end{array}\right)$ une matrice à coefficients entiers telle que

$$
\alpha \delta-\beta \gamma=1,\left(\begin{array}{ll}
\alpha & \beta \\
\gamma & \delta
\end{array}\right) \equiv\left(\begin{array}{cc}
p^{-1} & 0 \\
0 & p
\end{array}\right) \quad \bmod N
$$

et $\rho_{p}$ l'automorphisme de $\Re_{N}$ correspondant à $\left(\begin{array}{ll}p & 0 \\ 0 & p\end{array}\right)$. Alors, comme on a

$$
\left(\begin{array}{ll}
p & 0 \\
0 & p
\end{array}\right) \equiv\left(\begin{array}{ll}
p^{2} & 0 \\
0 & 1
\end{array}\right)\left(\begin{array}{cc}
\alpha & \beta \\
\gamma & \delta
\end{array}\right) \quad \bmod . N
$$

on voit que

$$
\boldsymbol{g}^{\rho p}(\tau)=\boldsymbol{g}\left(\frac{\alpha \tau+\beta}{\gamma \tau+\delta}\right) \text { pour } \boldsymbol{g} \in \mathbb{R}_{N}
$$

D’après la définition de $Y_{p}$, on peut donc regarder $M^{d}\left(\eta_{p}\right)$ comme une représentation de l'opérateur $R_{p}$ dans [4]. 


\section{§5. Résultats principaux.}

19. Les notations étant celles de $\mathbf{1 3}$, soient $g$ le genre de $\Gamma$ et $M_{l}$ une représentation $l$-adique de $\mathcal{A}(J)$. Si $l \neq p$, on peut choisir une représentation $l$-adique $M_{l, p}$ de $\mathcal{A}(J(p))$ de telle façon que, pour chaque $\mu \in \mathcal{A}(J)$, on ait $M_{l}(\mu)=M_{l, p}(\mu(p))$. Nous désignerons $M_{l, p}$ aussi par $M_{l}$. On a alors d'après $\left(\mathrm{I}^{\prime}\right)$

$$
M_{l}\left(\xi_{p}\right)=M_{l}(\pi)+M_{l}\left(\pi^{\prime} \eta_{p}\right) .
$$

Comme on a $\pi \pi^{\prime}=p \delta_{J}, U$ étant une indéterminée, on a

$$
I_{2 g}-M_{l}\left(\xi_{p}\right) U+M_{l}\left(\eta_{p}\right) p U^{2}=\left[I_{2 g}-M_{l}(\pi) U\right]\left[I_{2 g}-M_{l}\left(\pi^{\prime} \eta_{p}\right) U\right]
$$

où $I_{m}$ désigne la matrice unité de $m$ lignes. Selon que $J$ est égal à $J_{N}$ ou à $J_{N, \hat{l}}$, on a en vertu de $\left(\mathrm{II}^{\prime}\right)$

$$
I_{2 g}-M_{l}\left(\pi^{\prime} \eta_{p}\right) U=M_{l}(\alpha)^{-1}\left[I_{2 g}-M_{l}\left(\pi^{\prime}\right) U\right] M_{l}(\alpha)
$$

ou

$$
I_{2 g}-M_{l}\left(\pi^{\prime} \eta_{p}\right) U=M_{l}(\beta)^{-1}\left[I_{2 g}-M_{l}\left(\pi^{\prime}\right) U\right] M_{l}(\beta) .
$$

D’après la formule $M_{l}\left(\pi^{\prime}\right)=E_{l}(\Theta)^{-1 t} M_{l}(\pi) E_{l}(\Theta)$ dans [11] $\mathrm{n}^{\circ} 76$, on a

$$
\operatorname{det}\left[I_{2 g}-M_{l}\left(\xi_{p}\right) U+M_{l}\left(\eta_{p}\right) p U^{2}\right]=\operatorname{det}\left[I_{2 g}-M_{l}(\pi) U\right]^{2}
$$

pour $\Gamma_{N}$ ou $\Gamma_{N, \mathfrak{h}}$. Soit $M^{d}$ une représentation de $\mathcal{A}(J)$ par les formes différentielles de première espèce; alors $M_{l}$ est équivalent à $M^{d} \oplus \bar{M}^{d}$ où $\bar{M}^{d}$ désigne la représentation imaginaire conjuguée de $M^{d}$. Comme $J_{N}, J_{N, \mathfrak{h}}, \xi_{p}, \eta_{p}$ sont définis par rapport à $\boldsymbol{Q}$, on peut choisir la représentation $M^{d}$ de telle façon que $M^{d}\left(\xi_{p}\right)=\bar{M}^{d}\left(\xi_{p}\right)$ et $M^{d}\left(\eta_{p}\right)=\bar{M}^{d}\left(\eta_{p}\right)$; on a donc

et par suite

$$
\operatorname{det}\left[I_{2 g}-M_{l}\left(\xi_{p}\right) U+M_{l}\left(\eta_{p}\right) p U^{2}\right]=\operatorname{det}\left[I_{g}-M^{d}\left(\xi_{p}\right) U+M^{d}\left(\eta_{p}\right) p U^{2}\right]^{2}
$$

$$
\operatorname{det}\left[I_{2 g}-M_{l}(\pi) U\right]=\operatorname{det}\left[I_{g}-M^{d}\left(\xi_{p}\right) U+M^{d}\left(\eta_{p}\right) p U^{2}\right] .
$$

Désignons par $\zeta(s, \Gamma, p)$ la fonction $\zeta$ de $\Gamma(p)$; alors on a

$$
\zeta(s, \Gamma, p)=\left(1-p^{-s}\right)^{-1}\left(1-p^{1-s}\right)^{-1} \operatorname{det}\left[I_{g}-M^{d}\left(\xi_{p}\right) p^{-s}+M^{d}\left(\eta_{p}\right) p^{1-2 s}\right] .
$$

La fonction $\zeta$ de la courbe $\Gamma$ est définie par

$$
\zeta(s, \Gamma)=\prod_{p} \zeta(s, \Gamma, p),
$$

où le produit est étendu à tous les nombres premiers pour lesquels $\Gamma$ n'a pas de défaut. Dans $\mathbf{1 8}$, on a vu que $M^{d}\left(\xi_{p}\right), M^{d}\left(\eta_{p}\right)$ ne sont autres que les représentations des opérateurs $T_{p}, R_{p}$ pour les formes paraboliques de degré 2 (et d'espèce $N$ ).

Nous sommes ainsi parvenus à notre résultat principal:

La fonction $\zeta$ de la courbe $\Gamma_{N}$ s'exprime sous la forme 


$$
\begin{aligned}
\zeta\left(s, \Gamma_{N}\right) & =f(s) \zeta(s) \zeta(s-1) \Phi(s)^{-1}, \\
\Phi(s) & =\prod_{t, \varepsilon} \operatorname{det} \Phi_{t, \varepsilon}(s)
\end{aligned}
$$

où $\zeta(s)$ est la fonction $\zeta$ de Riemann, $f(s)$ est une fonction rationnelle de $p^{-s}$ et $\Phi_{l, \varepsilon}(s)$ désigne le produit d'Euler introduit par E. Hecke [4], attaché aux formes paraboliques de degré 2 , de diviseur $t$ et de caractère $\varepsilon(n)$.

On obtient un résultat analogue aussi pour la fonction $\zeta$ de $\Gamma_{N, h}$; dans ce cas, il faut seulement limiter $(t, \varepsilon)$ à certaines valeurs selon $\mathfrak{h}$ dans le produit pour $\Phi(s)$. Le cas où $\mathfrak{h}$ a l'index 1 ou 2 a été traité par M. Eichler [3].

20. Nous allons maintenant étudier les racines caractéristiques de $T_{p}$. Elles sont les racines de l'équation caractéristique de $M_{l}\left(\xi_{p}\right)=M_{l}\left(\tilde{\xi}_{p}\right)$ et par suite les racines caractéristiques d'une représentation régulière de $\tilde{\xi}_{p}$ dans l'algèbre $\mathscr{A}_{0}\left(\tilde{J}_{N}\right)$. Soit $\mathscr{B}$ l'algèbre sur $\boldsymbol{C}$ déduite de $\mathscr{A}_{0}\left(\tilde{J}_{N}\right)$ par extension à $\boldsymbol{C}$ du corps de base. Soit $\sigma$ le prolongement dans $\mathcal{B}$ de la trace de $\mathcal{A}_{0}\left(\tilde{J}_{N}\right)$ (cf. [10] p. 58); $\sigma\left(\mu^{\prime} \mu\right)$ est une forme d'Hermite positive non-dégénérée dans $\mathscr{B}$; posons $\|\mu\|=\sigma\left(\mu^{\prime} \mu\right)^{1 / 2}$ pour $\mu \in \mathscr{B}$. D'après les relations $\pi \pi^{\prime}=\pi^{\prime} \pi=p \delta$ et $\tilde{\alpha}^{\prime}=\tilde{\alpha}^{-1}$, on a

$$
\|\pi \mu\|=\sqrt{\bar{p}}\|\mu\|, \quad\left\|\left(\tilde{\alpha}^{-1} \pi^{\prime} \tilde{\alpha}\right) \mu\right\|=\sqrt{\bar{p}}\|\mu\| ;
$$

par suite, d'après $\left(\mathrm{I}^{\prime}\right),\left(\mathrm{II}^{\prime}\right)$ on a

$$
\left\|\tilde{\xi}_{p} \mu\right\| \leqq\|\pi \mu\|+\left\|\left(\tilde{\alpha}^{-1} \pi^{\prime} \tilde{\alpha}\right) \mu\right\|=2 \sqrt{\bar{p}}\|\mu\| .
$$

Il en résulte que les valeurs absolues des racines d'une représentation régulière de $\tilde{\xi}_{p}$ dans $\mathcal{A}_{0}\left(\tilde{J}_{N}\right)$ ne dépassent pas $2 \sqrt{p}$. Nous obtenons ainsi le résultat suivant:

Les valeurs absolues des racines caractéristiques de l'opérateur $T_{p}$ de Hecke pour les formes paraboliques de degré 2 ne dépassent pas $2 \sqrt{p}$ pour presque tous les nombres premiers $p$.

Université de Tokyo.

\section{Bibliographie}

[1] W.L. Chow, The jacobian variety of an algebraic curve, Amer. J. Math., 76 (1954), pp. 453-476.

[2] M. Deuring, Die Typen der Multiplikatorenringe elliptischer Funktionenkörper, Abh. Math. Sem. Univ. Hamburg, 14 (1941), pp. 197-272.

[3] M. Eichler, Quaternäre quadratische Formen und die Riemannsche Vermutung für die Kongruenzzetafunktion, Arch. Math., 5 (1954), pp. 355-366.

[4] E. Hecke, Über die Modulfunktionen und die Dirichletschen Reihen mit Eulerschen Produktentwicklung I, II, Math. Ann., 114 (1937), pp. 1-28, 316-351. 
[5] G. Shimura, Reduction of algebraic varieties with respect to a discrete valuation of the basic field, Amer. J. Math., 77 (1955), pp. 134-176.

[6] G. Shimura, On complex multiplications, Proc. International Symposium on algebraic number theory, Tokyo-Nikko, (1955), pp. 23-30.

[7] Taniyama, Jacobian varieties and number fields, ibid. pp. 31-45.

[8] G. Shimura et Y. Taniyama, Complex multiplication of abelian varieties and its applications to number theory, à paraître dans J. Math. Soc. Japan.

[9] A. Weil, Foundations of algebraic geometry, New York, (1946).

[10] A. Weil, Sur les courbes algébriques et les variétés qui s’en déduisent, Paris, (1948).

[11] A. Weil, Variétés abéliennes et courbes algébriques, Paris, (1948).

[12] A. Weil, On algebraic groups and homogeneous spaces, Amer. J. Math., 77 (1955), pp. 493-512. 